Chapter 14

\title{
A Systematic Approach to Visual System Neurorehabilitation - Population Receptive Field Analysis and Real-time Functional Magnetic Resonance Imaging Neurofeedback Methods
}

\author{
T. Dorina Papageorgiou, Amalia Papanikolaou and \\ Stelios M. Smirnakis
}

Additional information is available at the end of the chapter

http://dx.doi.org/10.5772/57000

\section{Introduction: Is visual rehabilitation inside a cortical scotoma possible, in principle?}

Visual information transmission flows from the retinal ganglion cells to the lateral geniculate nucleus and then to the primary visual cortex (V1), the chief cortical relay of visual information and in turn, to "higher" extrastriate areas. Beyond area V1, visual processing is distributed across multiple interconnected brain areas, the precise role of which and their interactions are not yet, completely understood. To add to the dynamic complexity of the system, feedback from higher areas and modulation by top-down processes, such as attention are often critical in the formation of visual percepts (Deco and Lee; 2004; Olhausen, 2003; Kastner and Ungerleider, 2000; Mumford, 1994; Hubel and Weisel, 1977).

Impairment of visual function can occur at any point along the visual pathway from the eye to the cortex. We focus our discussion here on V1 lesions, which result in dense contralateral visual field defects known as "scotomas". Scotomas as a consequence of area V1 lesions often involve the contralateral half of the visual field resulting in hemianopia, or a contralateral visual field quadrant the consequence of which is quadrantanopia. V1 lesions are the most prevalent injury of the visual cortex, often occurring as a result of posterior cerebral artery (PCA) stroke, hemorrhage, or traumatic brain injury (TBI) (Pambakian and Kennard, 1997; Zhang et al., 2006; Ajina and Kennard, 2012). Twenty to thirty percent of stroke survivors experience visual disability (Taylor, 1997; Gilhotra et al., 2002; Giorgi et al., 2009), while the 
incidence of significant visual perceptual impairment in TBI victims exceeds $50 \%$ in some studies (McKenna et al., 2006; Lew et al., 2007; Elisevich et al., 1984). The loss of visual perception inside a large scotoma can significantly affect the patient's ability to perform daily tasks, navigate in unknown environments, and function independently (Ajina and Kennard, 2012; Rizzo and Robin, 1996; Riggs, et al., 2007). Visual rehabilitation is clearly necessary for the daily living function of these patients. The literature describing visual rehabilitation efforts is extensive and doing justice to it all is beyond the scope of this chapter. We should mention at the outset that we will not discuss, the large literature on practicing eye movement strategies or, using prisms to remap the unseen onto the seen part of the visual field. Instead, we focus on novel approaches that aim to enhance perception inside the visual field scotoma.

To date, no established method exists to rehabilitate visual perception in adult patients with lesions of the primary visual cortex. The lack of effective methods for rehabilitation has led to the general perception that the adult visual cortex has decreased capacity to compensate after injury. This engenders diminished hope that successful strategies can be established to promote the recovery of visual perception after cortical injury. This is partly justified, as several attempts claiming to have achieved significant results have failed. The most notable recent example is an effort by Nova Vision claiming that a rehabilitative paradigm based on a "saccade-to-target" task could significantly shrink dense visual field scotomas (Kasten et al.; 1998, 1999; 2000, 2001, 2006; Poggel et al., 2001, 2004, 2006; Sabel et al., 2000, 2004; Werth and Moehrenschlager, 1999; Zihl and VonCramon, 1979; Zihl and Von Carmon, 1985; Jobke et al., 2009). Albeit early psychophysical training methods and Nova Vision studies were seen as promising (Kerkhoff et al., 1992; Kerkhoff et al., 1994; Julkunen, 2003; Kasten et al., 1995; 1998a, b, 1999; 2000, 2001; Poggel et al., 2001, 2004, 2006; Sabel et al., 2000, 2004; Werth and Moehrenschlager, 1999; Zihl and VonCramon, 1979; Zihl, 1990), later studies implementing rigorous eye movement controls failed to find a reduction in the visual field scotoma in patients with V1 lesions (Reinhard et al., 2005; Horton, 2005a; Horton, 2005b; Pleger et al., 2003).

Although these efforts are disappointing, the rehabilitation of scotomas resulting from V1 injuries is not altogether a cause without hope (Kasten et al., 1998; Huxlin et al., 2009; Schmid et al., 2010; Poggel et al., 2010; Sahraie et al., 2010; Schmid et al., 2009; Alexander and Cowey, 2009). On one hand, patchy injuries to area V1 or its inputs in the optic radiation seem to be amenable to rehabilitation, as training can help recruit and strengthen surviving connections. In support of this, Sabel and colleagues (Kasten et al., 1999) showed that $74 \%$ of patients with partial optic nerve involvement showed significant recovery with training, compared to $29 \%$ of patients with post-chiasmatic lesions. This is likely the result of increased recruitment of partially-lesioned fiber pathways or islands of residual vision (Fendrich et al., 1992). On the other hand, recent evidence suggests that even when lesions to area V1 or, its proximal inputs are dense, it may be possible to some extent to functionally bypass the area of the injury:

First, there exist anatomical pathways that bypass the area of V1 injury (fig. 1). One such pathway projects from the retina to the koniocellular (intercalated) layers of the lateral geniculate nucleus directly, or to the superior colliculus and from there to extrastriate cortex. This pathway originates in the retinal P $\gamma$ class of ganglion cells, which comprises $\sim 10 \%$ of the total ganglion cell number is particularly dense near the fovea (Henry and Reid, 2000), and is 
known to survive retrograde degeneration following V1 lesions (Cowey and Stoerig, 1989). Another V1-bypassing pathway projects from the retina to the pulvinar directly or via the superior colliculus and from there to the extrastriate visual areas. Notably, although parvocellular and magnocellular projections to the lateral geniculate nucleus and beyond markedly atrophy following striate cortical lesions (Vanburen, 1963; Mihailovic et alo., 1971), superior collicular (Dineen et al., 1982) and pulvinar (Cowey, 1974) projections remain unchanged.

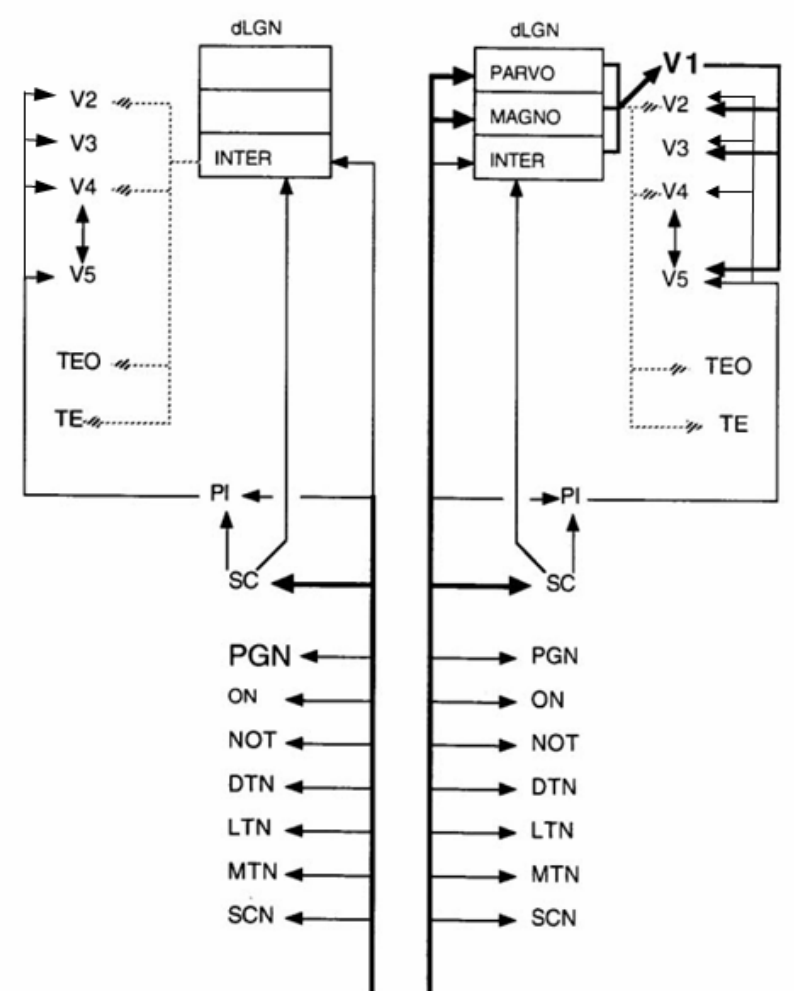

Figure 1. Overview of relevant anatomical visual pathways (Modified with permission from Stoerig and Cowey, 1997): Possible extra-geniculostriate pathways contributing to blindsight behavior, and residual extrastriate cortex activity, adapted from Stoerig and Cowey (Stoerig and Cowey, 1997). This diagram shows known retinofugal inputs, and some of the subsequent projections. On the right, the pathways are shown with intact V1. On the left V1 has been lesioned. Two pathways stand out as potentially mediating the residual activity observed in extrastriate cortex (Rodman et al., 1989; Schmid et al., 2009; Schmid et al., 2010), as well as related aspects of blindsight behavior: 1) The koniocellular pathway (dotted lines) from the $\mathrm{K}$ (intercalated) layers of the thalamus directly to areas V2, V3, V4, V5/MT. This pathway originates in the retinal Py class of ganglion cells, comprising $\sim 10 \%$ of total ganglion cells, survives retrograde degeneration following V1 lesions, and is particularly dense near the fovea (Cowey and Stoerig, 1989; Henry and Reid, 2000). This pathway receives both direct retinal and superior collicular input. 2) The projection from the inferior pulvinar to V2, V3, V4, V5/MT, which also receives direct input from the retina, as well as input from the retinotectal (superior colliculus) pathway. PGN: pre-geniculate nucleus, ON: olivary nucleus, NOT: nucleus of optic tract, MTN, LTN, DTN: medial, lateral, dorsal terminal accessory optic nuclei, SCN: suprachiasmatic nucleus, SC: superior colliculus, PI: inferior pulvinar. 
Second, these pathways have been shown to be functional under certain conditions. Lesions of area V1 or its post-chiasmatic afferents deprive the extrastriate visual cortex of its main input and result in a dense contralateral visual field scotoma, in which conscious visual perception is thought to be irreversibly lost (Cowey and Stoerig, 1991; Stoerig and Barth, 2001). Remarkably, despite the absence of a conscious visual percept, a capacity to process certain attributes of the visual stimulus persists inside the scotoma, the phenomenon known as "blindsight" (Kluver, 1936; Poppel et al., 1973; Weiskrantz, 1974). The blindsight phenomenon implies that at least some extra-geniculo-striate retinofugal pathways (Cowey, 2010; Schmid et al., 2010; Weiskrantz, 2004; Schoenfeld et al, 2002; Moore et al., 2001; Goebel et al., 2001; Stoerig and Cowey, 1997; Moore et al., 1995; Cowey and Stoerig, 1991; Girard et al., 1991; Pasik and Pasik, 1971) can functionally bypass area V1. This is corroborated by experiments in humans and primates, which have directly demonstrated that extrastriate areas can be modulated by the visual stimulus in the absence of V1 input (Rodman et al., 1989; Baseler et al., 1999; Goebel et al., 2001; Schmid et al., 2009; Schmid et al., 2010). For example, Rodman and Gross demonstrated that area V5/MT can be directly activated through the pathway bypassing area V1 via the superior colliculus (Rodman et al., 1989; Rodman et al., 1990), while Schmid et al. (Schmid et al., 2009; Schmid et al., 2010) showed that early extrastriate areas V2, V3 can be visually modulated by the LGN in the absence of V1 input. Schmid et al. further elucidated that transiently inactivating LGN in V1 lesioned animals not only abolishes visual modulation in areas V2, V3 but also, returns the monkey's blindsight performance to chance. Unfortunately, the V1-bypassing pathways that mediate the blindsight phenomenon are weak and of limited practical value. The potential of these pathways to induce recovery remains unrealized. This underscores the need to examine the mechanisms underlying the recovery reported in recent studies (Huxlin et al., 2008; Huxlin et al., 2009; Sahraie et al., 2006) in order to understand how to develop effective rehabilitative paradigms. It remains to be examined whether novel neuro-rehabilitative training algorithms can strengthen V1-bypassing pathways to derive practical benefit.

Third, training can improve performance inside the scotoma of subjects with area V1 lesions. Behavioral training in healthy subjects can improve visual performance by inducing plasticity and reorganization in the physiology of visual networks (Karni and Sagi, 1991; Liu et al., 2000; Yang and Maunsell, 2004; Ahissar and Hochstein, 1997). Perceptual learning is retinotopically specific, suggesting it involves a use-dependent synaptic enhancement induced by pre-and postsynaptic activity (Brown et al., 1988). Studies in humans (Pleger et al., 2003; Taub et al., 2002; Weiller, C. 1998; Lindberg et al., 2003, Takeuchi et al., 2005) and animals (Rudolph et al., 1994; Rudolph and Pasternak, 1999; Rudolph and Delay, 1993, Fabre-Thorpe et al., 1994; Friel et al., 2000; Huxlin and Pasternak, 2004) with V1 lesions, as well as behavioral studies of "blindsight" (Chokron et al., 2008; Stoerig and Cowey, 1997; Sahraie et al., 2006; Overgaard, 2011) suggest that visual performance in the scotoma can also improve with training (Raninen et al., 2007; Henriksson et al., 2007). More recently, Huxlin et al. (2006)-following their work on cats-trained V1+lesioned patients to perform a two-alternative forced choice random dot kinematogram (RDK) direction of motion 
discrimination task in their blind hemifield (Huxlin et al., 2009). Remarkably, direction of motion discrimination thresholds recovered from chance to normal at trained locations (Huxlin et al., 2009). Eye movements were strictly controlled, and there were no obvious artifacts that could confound the findings. Recovery was retinotopically specific, but could be extended by training consecutively adjacent locations that lay progressively deeper inside the scotoma, inducing recovery up to $\sim 20^{\circ}$ from the scotoma border in one subject. Furthermore, recovery in this task appeared to carry some practical significance, as the subjects' ability to dodge basketballs "thrown" at them from the blind hemifield in a virtual reality environment, improved (Iorizzo et al., 2011). These findings sparked renewed interest in studying visual rehabilitation strategies. This is encouraging, but it is necessary to note that visual rehabilitation results appear more variable across the literature (see table 1 , in a recent review by Sabel et al. (Sabel et al., 2011), and (Horton, 2005a; Horton, 2005b) for a critical review of the field). Three important questions remain to be answered regarding scotomas resulting from V1 lesions: i) can visual rehabilitative training result in improved visual performance of practical significance? ii) what is the underlying mechanism of recovery? and 3) what is the optimal method for visual rehabilitative training?

In summary, even though visual rehabilitation following area V1+lesions is a difficult problem, it is not a hopeless endeavor. Anatomical pathways bypassing the area of the lesion exist, and they have been demonstrated to be functional in certain situations. Although early trials have been inconclusive, a recent report by Huxlin et al. (Huxlin et al., 2009), suggests that some recovery is possible, at least in the domain of visual motion perception. Further studies are needed: (i) to independently corroborate the results of Huxlin et al. (Huxlin et al., 2009); (ii) to understand what visual attributes and types of lesions are amenable to recovery; and (iii) to study the mechanism of recovery. It is important to note that we do not expect even successful rehabilitation methods to restore vision to pre-lesion levels. For one, the quality of the restored visual percept will most likely differ from normal. The reason is that, following V1 lesions, the magnocellular and parvocellular pathways largely degenerate (fig. 1), shifting the balance towards the koniocellular pathway, which is spared. Nevertheless, successful visual rehabilitation can train patients to use the qualitatively and quantitatively different form of visual perception mediated by appropriately strengthened V1-bypassing pathways. The design of such a neuro-rehabilitative approach will confer considerable practical significance.

\section{Using fMRI to functionally characterize and classify cortical lesions and corresponding scotomas}

To design effective visual rehabilitation strategies for cortical scotomas, we have to grapple with the issue of lesion variability. Cortical lesions differ from individual to individual, and this impacts whether or not the resulting scotoma is amenable to rehabilitation. Consequently, some patients show good recovery following visual rehabilitative training (Kasten et al., 
1998; Huxlin, 2009) and others no recovery at all (Reinhard et al., 2005; Horton et al., 2005a; Horton et al., 2005b; and our personal observation). It remains unclear what criteria one may use to select patients more likely to recover. Scotomas are mapped using visual field perimetry to determine the part of the visual field where visual perception is impaired. A problem faced in studies of visual rehabilitation is that patients often have heterogeneous lesions, even though the extent and density of their perceptual visual scotomas, measured by perimetry, match. Conversely, the anatomical characterization of the lesion is not always a reliable indication of the properties or, the extent of the resulting scotoma. Consequently, neither visual field perimetry maps nor, purely anatomical information are sufficient indicators of the capacity for rehabilitation.

A measure of the ability of visual stimuli presented inside the scotoma to elicit perceptually sub-threshold activity in spared visual cortex would add valuable information. Functional magnetic resonance imaging (fMRI) can be used to identify which sectors of the visual field scotoma remain able to transmit visual information to spared regions of the visual cortex (fig. 3), downstream from the lesion. This can help to classify functionally different types of lesions that yield similar scotomas, and to identify regions of the scotoma that elicit different patterns of functional activation and may therefore, have different capacity for rehabilitation. The underlying hypothesis is that parts of the scotoma that can still convey visual information to higher areas, bypassing the cortical lesion, will be more amenable to rehabilitation. Moreover, the extrastriate areas that become activated may reveal clues about the attributes of the visual stimulus that will be more amenable to rehabilitation.

We propose to apply state-of-the-art fMRI methods to characterize voxel by voxel, how population receptive fields (pRF; Wandell et al., 2007; Dumoulin and Wandell, 2008; Amano et al., 2009; Lee et al., 2013) in spared visual areas are organized to cover the visual field following cortical visual pathway injuries (see figs. 2, and 3) (Baseler et al., 2011). The pRF of a voxel refers to the region of visual space that elicits a visually-induced modulation of the BOLD (Blood-Oxygen-Level-Dependent) signal in that voxel. Various pRF models have been proposed in the literature (Dumoulin and Wandell, 2008; Zuiderbaan et al., 2012; Lee et al., 2013). The simplest and most commonly used is a circularly symmetric, 2D Gaussian model with center $(x, y)$ and radius $(\sigma)$ (Dumoulin and Wandell, 2008). The BOLD time series predicted by this model is derived by convolving the pRF model with the stimulus sequence and the BOLD hemodynamic response function (HRF; Boynton et al., 1996; Worsley et al., 2002). The $\mathrm{pRF}^{\prime} \mathrm{s}$ parameters are then estimated by fitting the BOLD signal predicted by the model to the actual BOLD signal measurement obtained from each voxel.

Early methods of retinotopic mapping, such as ring and wedge stimuli (DeYoe et al., 1996; Dougherty et al., 2003; Engel et al., 1994; Sereno et al., 1995), as well as moving bar stimuli (Wandell et al., 2007), which traverse the visual field in different directions can usually provide robust pRF estimates (see fig. 2) rendering them useful for studying cortical reorganization. One limitation of direct-fit pRF estimation methods is that these can result in estimation biases at the scotoma border (Lee et al., 2013). Recently, a 2-step pRF estimation method based on first estimating the $\mathrm{pRF}$ topography, thresholding it, and then fitting an appropriate $\mathrm{pRF}$ model 

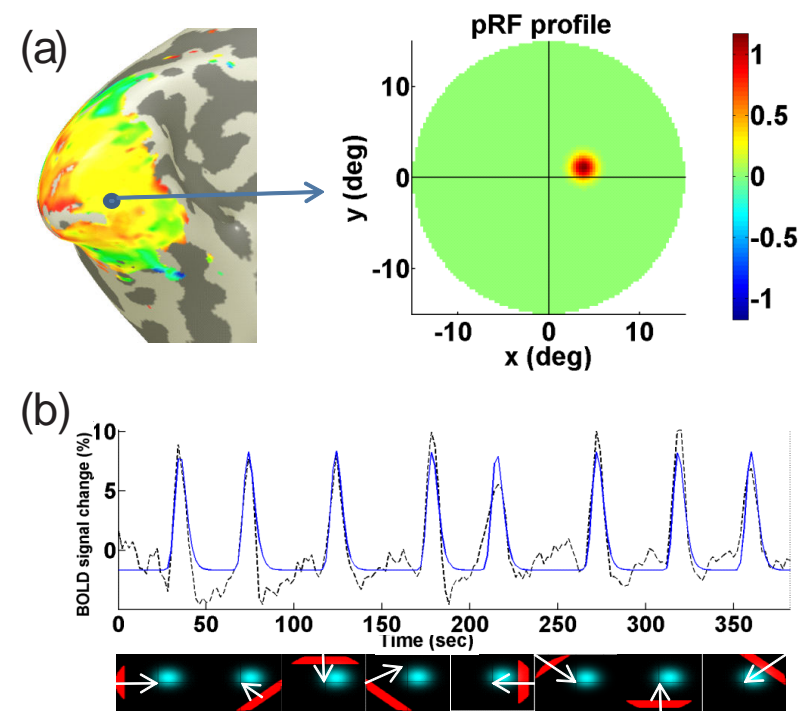

Figure 2. The population receptive field (pRF) model. This model estimates the region of the visual field that elicits a response in a small region (voxel) of the visual cortex. One implementation of the pRF model is a circularly symmetric Gaussian receptive field in visual space whose center and radius are estimated by fitting the BOLD signal responses to the estimated responses elicited by convolving the model with the moving bar stimulus and the hemodynamic response. (a) shows the estimated position and size of the pRF in the visual field of a voxel located in V1. (b) shows the BOLD time-series (dashed line) and the model prediction (solid line) from the same voxel. The model explains a large amount of variance in the time course data. Below, we illustrate the position and direction of motion of the stimulus bar that elicited the peaks in the BOLD signal.

has been introduced to largely circumvent this problem (Lee et al., 2013). This is the preferred method to use near the scotoma border.

\subsection{Visual field coverage maps can potentially help to guide neurorehabilitation strategies}

Population receptive field analysis can measure the residual capacity of areas controlling vision to process visual information following V1+injuries. Plotting the pRFs from all voxels of a given area together as a color map reveals the "visual field coverage map" of that area. This represents the part of the visual field that can visually modulate the area. Note that visual field coverage maps of extrastriate areas often overlap with the area of the dense perceptual scotoma, measured by visual field perimetry. This is illustrated in the second panel of figure 3 for the human middle temporal cortex (hV5/MT+), an area important for visual motion perception (Zeki et al., 2004; ffytche et al., 2000; Zeki and ffytche et al., 1998). Note that the pRF maps of many hV5/MT+voxels lie inside the perceptual scotoma (left upper quadrant in fig. 3). In fact, in this specific case (fig. 3), they cover the whole extent of the scotoma. This implies that $\mathrm{hV} 5 / \mathrm{MT}+\mathrm{is}$ activated by visual stimuli presented in the left upper quadrant even though the subject does not perceive these stimuli. This suggests that there is a functional V1-bypassing pathway to area hV5/MT+that may promote recovery, if appropriately rehabilitated. There- 
fore, a promising rehabilitation strategy is to strengthen this pathway. It is likely, that it will be easier to rehabilitate visual motion perception inside parts of the scotoma that are covered by the pRF maps of area hV5/MT+. It is also, likely that rehabilitation will be even easier in parts of the scotoma that are also covered by the pRF maps of spared, earlier, visual areas. The third panel of fig. 3 illustrates the visual field coverage map of the spared portion of area V1. Note, that this extends above the horizontal meridian to partly overlap with the area of the scotoma. This defines two regions where visual rehabilitation may be different, according to the above hypothesis. The region of the scotoma indicated by the green arrow is represented in the coverage maps of both area hV5/MT+and the spared area V1, and is expected to have higher potential for rehabilitation. The region of the scotoma indicated by the blue arrow is represented only in the coverage map of area $\mathrm{hV5} / \mathrm{MT}+$ and is expected to present a more difficult challenge for rehabilitation. Visual field coverage maps (Amano et al., 2009) obtained by fMRI are an important adjunct to perimetric maps as they often provide complementary information (personal observation) and will likely be useful in tailoring therapy to appropriate visual field locations.
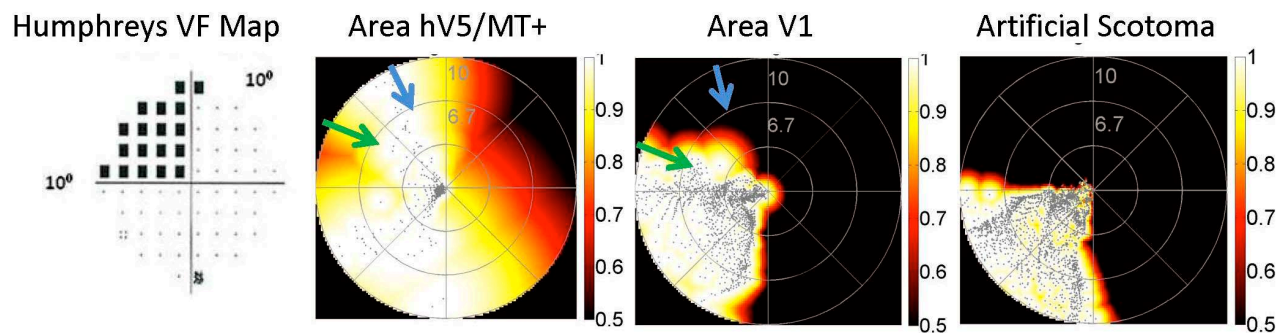

Figure 3. Visual Field Coverage Maps of spared visual areas with significant overlap with the region of the scotoma, define visual field locations that may be more amenable to rehabilitation. Humphreys VF Map panel: Humphreys 10-2 visual field map, illustrating that the subject had a dense left upper quadrantanopia. Area hV5/MT \pm panel: The visual field coverage map (Amano et al., 2009) of the right hV5/MT+shows visual field locations that evoke significant activity from hV5/MT+of the lesioned hemisphere. At each visual field location, the highest pRF value of all pRFs that cover this location is plotted. PRF normalization limits the range of values between $0-1$. Large values indicate significant visual modulation. Note, that although the subject is blind in the left upper quadrant, the subject's hV5/MT+responds to stimuli presented in the left upper quadrant. A potential advantage of using visual field coverage maps is that therapy can be individualized to each patient's appropriate visual field locations, which are not necessarily predictable from perimetric maps. Area V1 Panel: Visual field coverage map of the spared right area V1 extends above the left horizontal meridian into the dense area of the scotoma seen in the Humphreys map. This activity may be induced in orthotopic voxels that survive and are partially active following the V1 lesion (Kasten et al., 1998), or in anatomically ectopic V1 voxels that belong to the upper occipital lobe, which would ordinarily have had receptive fields in the left lower visual field quadrant. Note, that this area does not cover the entire quadrant, as is the case of the coverage map in area hV5/MT+. This mismatch will likely have implications for rehabilitation. For example, it may be easier to rehabilitate regions of the scotoma where the visual field coverage maps of spared V1 and hV5/MT \pm are congruent (green arrow), as opposed to incongruent (blue arrow). Control with AS (artificial scotoma) Panel: Visual field coverage map from the entire area V1 of a normal subject with an "artificial scotoma" simulating left upper quadrantanopia. By artificial scotoma we mean an area of the stimulus being excluded, in order to simulate the patient's scotoma. Note, that the visual field coverage map in this control case is as expected, i.e. it does not encroach into the left upper quadrant. 


\subsection{Quantitative $\mathrm{pRF}$ measurements provide a useful biomarker for gauging the effect of neurorehabilitation strategies}

Fig. 4 illustrates that pRF measurements in area hV5/MT+ipsilateral to a chronic V1+lesion differ from those in the normal hemisphere. Specifically, pRFs in hV5/MT+voxels of the lesioned hemisphere are smaller on average, and pRF-centers cluster near the vertical meridian ( $x=0$; fig. $4 \mathrm{C}$ ). Training may further change the pRF topography. Changes in the pRF topography before and after training can be a potentially useful biomarker for evaluating different rehabilitation paradigms before perceptual recovery becomes evident. Applying this approach systematically can help to formulate new hypotheses guiding future neuro-rehabilitation attempts. One hypothesis is that following visual motion rehabilitation training the sensitivity to motion stimuli of $\mathrm{hV} 5 / \mathrm{MT}+$ will increase, which will positively correlate with behavioral recovery. Alternatively, attentional networks or, other "higher" areas that receive input from hV5/MT+may reorganize to process visual motion information more effectively. Analyzing pRF maps obtained from visually responsive areas before and after training, will allow us to investigate the above hypotheses and to adopt appropriate rehabilitation strategies.

(A)

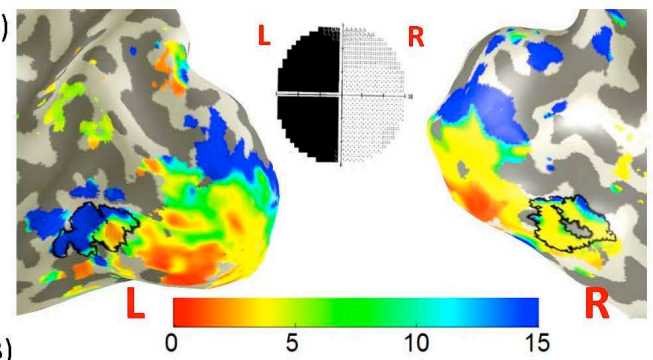

(B)

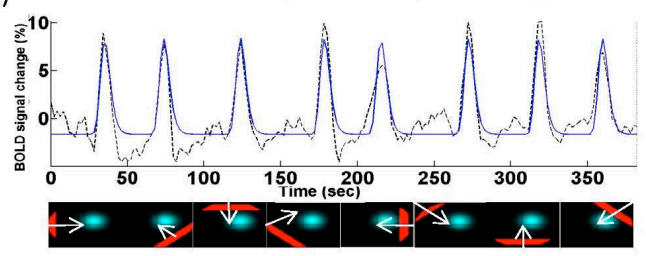

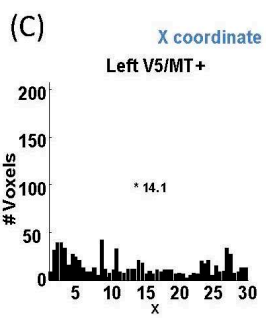

(D)

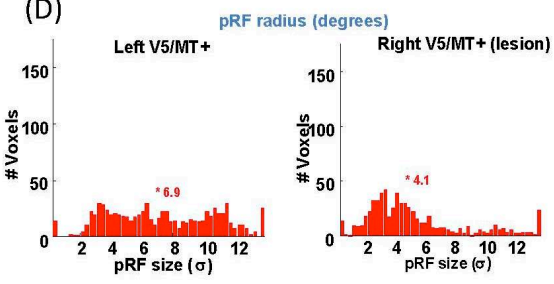

Figure 4. $h V 5 / M T \pm$ pRF mapping in a hemianopic patient: (A) Inflated occipital lobe of the normal (left) and lesioned (right) hemisphere with hV5/MT+outlined on the eccentricity map. Mid-inset is a 30-2 Humphrey perimeter showing dense left hemianopia. (B) Illustrates the method used to calculate pRF parameters (same as in fig. 2). The pRF model parameters are optimized to fit the BOLD signal, voxel by voxel. Note the close fit between the BOLD time-series (dashed line) and the pRF model prediction (solid line). The position and direction of motion of the stimulus bar that elicited each response is illustrated (bottom). (C) The X coordinate of the pRF centers in hV5/MT+of the normal (left) hemisphere is evenly distributed while in the lesioned (right) hemisphere it clusters near the vertical meridian ( $\mathrm{x}=0$ ). Note however, that the pRF centers still lie within the scotoma (negative $X$ values). (D): Distribution of pRF radii of $\mathrm{hV} 5 / \mathrm{MT}+$ voxels in the intact (left) and in the lesioned (right) hemisphere. The distribution of pRF radii shifts to smaller values in area hV5/MT+of the lesioned hemisphere. This may be because ipsilesional hV5/MT+voxels are driven by a V1-bypassing pathway that drives visual periphery less effectively. 
In summary, pRF measurements:

1. Classify subregions of the perimetric scotoma depending on how they are covered by spared visual areas; different regions will likely have different potential for rehabilitation.

2. Allow us to study the mechanism by which rehabilitation strategies improve visual performance.

3. Serve as quantitative biomarkers to evaluate the effects of training before perceptual recovery becomes evident, accelerating the pursuit of a rational strategy for visual rehabilitation.

Other methods of analysis can be applied here, but we do not have the space to do them justice. We mention briefly the promise of recent developments in effective connectivity analysis (Fuji et al., 2009; Stilla et al., 2008; Hinrichs et al., 2006) and diffusion tensor imaging (Wedeen et al., 2012; Yeatman et al., 2012; Van den Stock et al., 2011; Sherbondy et al., 2008; Fields, 2008; Okada et al., 2007; Schoth et al., 2006; Kikuta et al., 2006; Dougherty et al., 2005; Taoka et al., 2005; de Gelder et al., 2005; Reinges et al., 2004; Morris et al., 2001) for studying inter-area pathways that survive post-lesion, and whether they can become stronger by training. Population receptive field and effective connectivity analysis can be used to explore visual system reorganization and recovery following injury, and to generate concrete hypotheses on how to enhance and accelerate recovery of visual function by the application of cutting-edge rehabilitative strategies.

\section{How to approach visual neurorehabilitation?}

To date, we have little understanding of how the visual cortex reorganizes after injury, and no proven effective treatment strategies to rehabilitate the recovery of visual perception in the affected portion of the visual field in V1-lesioned patients. Understanding how to manipulate the brain's capacity for plasticity is an important step in the long-term effort to design treatments aiming to enhance the ability of the nervous system to recover after injury. To make progress along this front, we need to: i) study the mechanisms by which the adult brain adapts and reorganizes after injury; and ii) devise approaches that will allow us to manipulate the process of reorganization to induce visual recovery.

The network of visual areas can be viewed as a heavily interconnected circuit subject to a series of hierarchy rules. Early areas usually process sensory information initially, by passing it on to higher areas, and in turn, extract "higher" order features and control the flow of information through feedback loops. Increased performance following training can therefore be the result of changes that occur in early areas (Schoups et al., 2001; Yotsumoto et al., 2008; Censor and Sagi, 2009; Karni and Sagi, 2008), or the result of changes that occur in "higher" visual areas and attentional networks (Law and Gold, 2008; Yang and Maunsell, 2004; Lewis et al., 2009). Area V1 injuries, interrupt the cardinal feed-forward pathway but, as discussed above, visually driven information can still activate surviving extrastriate areas through bypassing routes (Cowey, 1974; Dineen et al., 1982; Rodman et al., 1989; Cowey and Stoerig, 1997; Baseler et al., 
1999; Goebel et al., 2001; Schmid et al., 2009; Schmid et al., 2010). The pattern of activity elicited in surviving visual areas interacts with higher "centers" in frontal, parietal and temporal areas but, in the absence of V1 input, fails to generate a strong visual percept. We suggest here two general, non-mutually exclusive approaches to visual rehabilitation:

"Bottom-up" approach: Visual rehabilitation strengthens V1-bypassing pathways to increase the response elicited in surviving extrastriate areas.

"Top-down" approach: Visual rehabilitation reorganizes higher "centers" to learn to process the modulated patterns of activity elicited in extrastriate areas by V1-bypassing inputs.

Non-invasive approaches to visual rehabilitation aim to enhance these pathways by recruiting the mechanisms of plasticity the brain uses for learning. Various behavioral approaches have been used. They usually involve performing a visual task that directs attention to a subthreshold stimulus, requires a choice, and then provides feedback about correct and incorrect choices (see fig. 5). Although such methods are effective for perceptual learning in general (Yotsumoto et al., 2008; Law and Gold, 2008; Yang and Maunsell, 2004), in the domain of rehabilitation of a dense perceptual scotoma results have been at best variable (Huxlin et al., 2009; Raninen et al., 2007 Sahraie, et al., 2006; Reinhard et al., 2005; Horton et al., 2005a; Horton et al., 2005b; Pleger et al., 2003). The most notable exception has been a recent well-controlled report by Huxlin and co-workers (Huxlin et al., 2009), which demonstrated strong recovery of direction of visual motion perception inside the scotoma of 5 hemianopic subjects (Huxlin et al., 2009; Huxlin, 2006). Two other groups independently, Sahraie et al. and Raninen et al., report that visual sensitivity can improve with training in humans with homonymous scotomas (Sahraie, 2006; Henriksson et al., 2007; Raninen et al., 2007). Encouraging results were also obtained by Pleger and co-workers, who showed that visual cortex reorganization was possible via daily visual stimulation training over a period of 6 months in 3 subjects with partial cortical blindness (Pleger et al., 2003). Although these are encouraging reports, the issue remains far from settled (Horton et al., 2005a; Horton et al., 2005b). Additional studies corroborating recent results and probing the mechanism of recovery are clearly needed to guide the implementation of new, more effective rehabilitation strategies. In what follows, we discuss the promise and challenges of a new visual rehabilitation approach, which aims to use "real time" fMRI neurofeedback to train subjects to promote plasticity in V1-bypassing pathways relevant to recovery.

\section{Introduction to real-time functional Magnetic Resonance Imaging neurofeedback approaches}

Less than two decades ago, the real-time functional magnetic resonance (rt-fMRI) method has been introduced (Cox, 1995) in the field of neuro-rehabilitation, which extracts the BOLD (Blood-Oxygen-Level-Dependent) signal from the subject's brain in real-time and uses it to provide feedback to the subject. Since the BOLD signal reflects neural activity this approach is called real-time fMRI neurofeedback (rt-fMRI $\mathrm{nFb}$ ). Multiple studies have shown that rt$\mathrm{fMRI} \mathrm{nFb}$, can train subjects to modulate the magnitude and spatial extent of the activity 
elicited in various cortical and subcortical areas (Berman et al., 2011; Bray et al., 2007; Caria et al., 2007; Caria et al., 2010; Chiew et al., 2012; deCharms et al., 2004; deCharms et al., 2005; Frank et al., 2012; Haller et al., 2010; Hamilton et al., 2011; Hinds et al., 2011; Johnson et al., 2012; Johnson et al., 2011; Johnson et al; 2010; Lee et al., 2012; Li et al., 2012; McCaig et al., 2011; Papageorgiou et al., 2009a; Papageorgiou et al., 2009b; Papageorgiou et al., 2013; Posse et al., 2003; Rota et al., 2009; Ruiz et al., 2013; Scharnowski et al., 2012; Shibata et al., 2011; Subramanian et al., 2011; Sulzer et al., 2013; Veit et al., 2012; Weiskopf et al., 2007; Weiskopf et al., 2004; Weiskopf et al., 2003; Yoo and Jolesz, 2002; Yoo et al., 2008; Zotev et al., 2011). The goal of this approach is to train subjects to control the pattern of their brain activity in a way that promotes a desired behavior. It can also be used to boost the neural capacity for learning and plasticity (Shibata et al., 2012; Scharnowski et al., 2012; Shibata et al., 2011; Weiskopf et al., 2004; deCharms et al., 2004; deCharms et al., 2005). If this is applied effectively, it could serve as a useful tool to promote neuro-rehabilitation.

The ability of rt-fMRI nFb to induce a behavioral change was first shown by Weiskopf et al. (Weiskopf et al., 2003) and deCharms et al. (deCharms et al., 2005). In the deCharms et al. study, chronic-pain patients were coached to decrease their pain by learning to control the BOLD signal intensity of the rostral anterior cingulate cortex (rACC), a region known to be involved in pain perception (Apkarian et al., 2005; Peyron et al., 2000). After training, subjects were able to voluntarily increase or decrease the rACC BOLD signal intensity, which was correlated with an increased or decreased level of pain, respectively; i.e., a $50 \%$ decrease in the rACC activity of chronic pain subjects corresponded approximately to a $64 \%$ decrease in their pain. This effect was specific to rtfMRI nFb training applied to rACC; i.e., no effect was seen after similar training conducted without rtfMRI NFb or, after sham rtfMRI nFb training derived from the activity of another subject's rACC.

Similar results have been obtained in other cortical and subcortical domains (Posse et al., 2003; Lee et al., 2011; Hamilton et al., 2011; Ruiz et al., 2013). A recent study showed that healthy volunteers were able to volitionally regulate the activity of their insula when given rt-fMRI $\mathrm{nFb}$ (Lee et al., 2011). Posse and his team (Posse et al., 2003) trained subjects to upregulate their amygdala, an area whose level of activity is associated with sad affect and depression (Wang et al., 2012; Anand et al., 2007; Liu et al., 2011). Amygdala upregulation induced by rt-fMRI feedback was positively correlated with self-ratings of sadness across repeated fMRI sessions (Posse et al., 2003). Conversely, Hamilton et al. used rt-fMRI nFb to train subjects to downregulate subgenual ACC and posterior cingulate cortex, resulting in positive mood induction (Hamilton et al., 2011). Sham rt-fMRI nFb showed no effect. Ruiz et al. showed that schizophrenic patients can be trained by rt-fMRI $\mathrm{nFb}$ to voluntarily control their anterior insula bilaterally (Ruiz et al., 2013). The effect of bilateral anterior insula activation is reflected on their ability to recognize face emotion, a known deficit in schizophrenia. These findings collectively, suggest that: 1) rt-fMRI nFb can be used to train subjects to voluntarily control specific areas; 2 ) changes in the activity of certain areas can be associated with significant behavioral changes; 3) $r t-f M R I n F b$ training can achieve stronger behavioral results than similar training without $n F b$; and 4) rt-fMRI training can induce reorganization that can outlast the period of the training inside the magnet and even induce visual perceptual learning (Shibata et al. 2011). 
Shibata et al. in a seminal study used rt-fMRI nFb to induce perceptual learning (Shibata et al., 2011). Rt-fMRI nFb methods were used to enable subjects to induce activity patterns in their early visual cortex corresponding to one particular orientation. Initially, the subjects performed an orientation discrimination task and a decoder of area V1/V2 activity was constructed to classify a pattern of the measured fMRI signals into one of three orientations. Once the decoder was constructed, each subject participated in a 5 to 10-day rt-fMRI nFb stage, during which they learned to induce patterns of activity in areas V1/V2 corresponding to the target orientation. During this stage, subjects were instructed to maximize the signal delivered to them via feedback, but were not told how to induce the desired patterns of activity that would result in increased activity. Subjects did not know what was to be learned. Using this strategy they were able to induce visual perceptual learning specific to the target orientation in areas V1/V2. Learning occurred as a function of the subject's ability to elicit the particular pattern of activation corresponding to the target orientation in early visual areas. Remarkably, subjects were able to generate this pattern simply by being given the instruction to maximize feedback, without being aware that the pattern to be elicited was related to orientation. This demonstrated that the rt-fMRI $\mathrm{nFb}$ method can be used to induce highly specific activity patterns within a brain region and that repeatedly eliciting the desired pattern of activity is sufficient to induce plasticity in early visual areas. These findings suggest that $r t-f M R I n F b$ training can be used to induce targeted and individualized plasticity in the visual system.

The studies described above (Ruiz et al., 2013; Linden et al., 2012; Subramanian et al., 2011; Shibata et al., 2011; Lee et al., 2011; Hamilton et al., 2011; deCharms et al., 2004; Posse et al., 2003) show that manipulating the activity in select brain areas can induce plasticity. Modified paradigms can also be designed to increase plasticity along specific pathways, by co-activating input and recipient neuronal populations. For example, a projection from area A to recipient area $B$ in the brain can increase or, decrease in strength, depending on the relative activity between input projections from A and recipient neurons in B. Rt-fMRI nFb does not have the temporal resolution necessary to precisely implement Hebbian mechanisms of plasticity. Nevertheless it can be used to train the subject to voluntarily manipulate the activity level of select neuronal populations in area B while their input from A is presented. The hypothesis is that "top-down" activation of area B enhanced by nFb judiciously paired with "bottom-up" presentation of inputs that activate A, can increase the strength of the projection A->B either by their interaction or additive effect. Below, we discuss an example outlining how this proposal might work for the rehabilitation of visual motion perception following area V1 lesions (see also fig. 5).

Area hV5/MT+is associated with global coherent visual motion perception. The goal is to use rt-fMRI $\mathrm{nFb}$ methods to strengthen the neural pathways bypassing the V1 lesion and project to area $\mathrm{hV} 5 / \mathrm{MT}+\mathrm{in}$ order to improve visual motion perception of random dot kinematogram (RDK) stimuli (see fig. 5). The first step is to train the subject to voluntarily upregulate their hV5/MT+activity. To do this, we ask the subject to practice mental imagery of fully coherent visual motion stimuli in their blind hemifield, moving in the direction of the anticipated stimulus. During mental imagery, the subject receives $\mathrm{rt}-\mathrm{fMRI} \mathrm{nFb}$ proportional to the activity in their hV5/MT+via a visual interface at fixation (red arrow in fig. 5). Subjects are trained to 


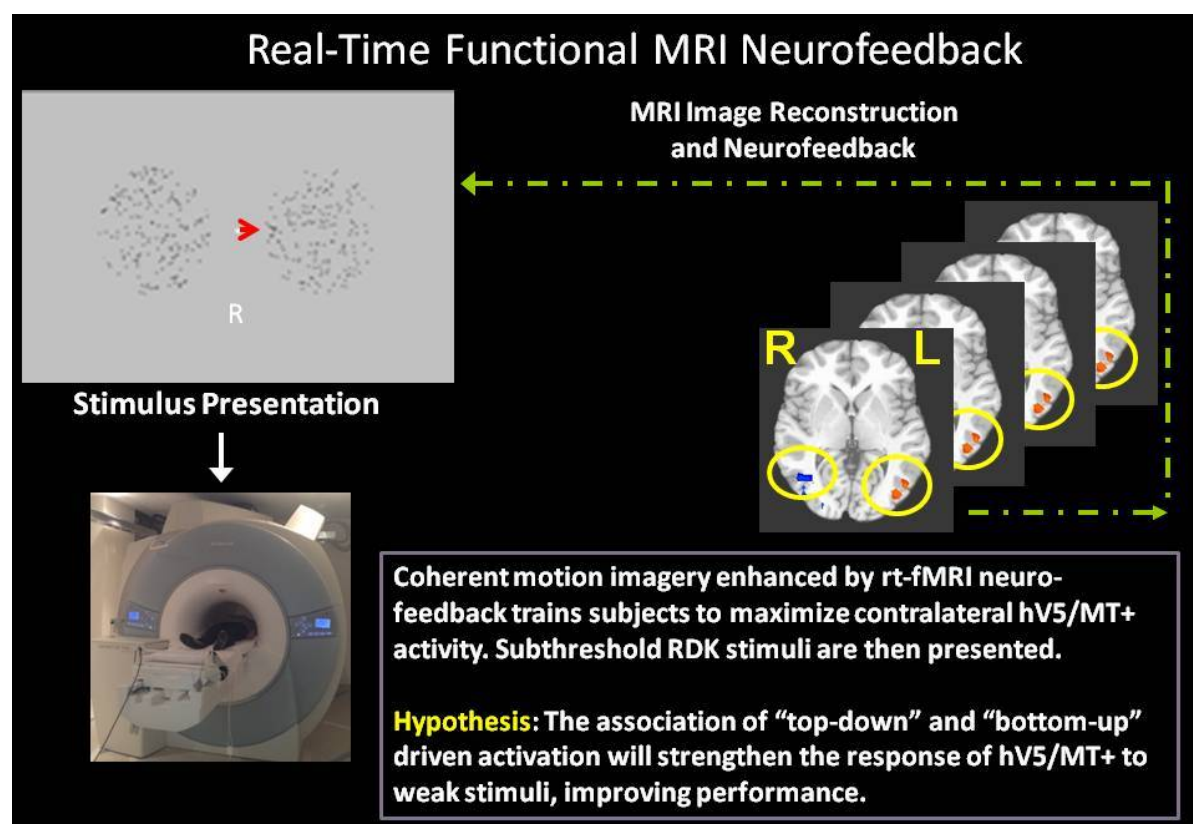

Figure 5. Rt-fMRI nFb paradigm for neurorehabiliation of visual motion perception: The subject lies supine inside the scanner, while s/he is presented with a $0 \%$ coherent RDK stimulus. Brain volumes are acquired every $2 \mathrm{sec}$ (TR). $\mathrm{hV} 5 / \mathrm{MT+}+$ circled) is selected as our ROI. Turbo Brain Voyager software (Brain Innovation) is used to deliver $\mathrm{nFb}$. The BOLD signal in hV5/MT+is estimated in real time (every TR), normalized, and "fed" back, via the length of a horizontal arrow at fixation, to train the subject to upregulate area hV5/MT+. Subjects are instructed to attend to the RDK they are cued towards, either right $(\mathrm{R})$ or left $(\mathrm{L})$ and imagine that it is coherent (when in fact it is not). This increases the level of activity in the contralateral hV5/MT+: (i) superimposing coherent motion via imagery on the right RDK increases left hV5/MT+activity, which is color-coded in red; (ii) superimposing coherent motion via imagery on the left RDK increases right hV5/MT+activity, which is color-coded in blue. The subject uses the length of the arrow to determine the degree of effort and success of his/her strategy. When the rt-fMRI nFb driven, "top-down," hV5/MT+activation crosses a threshold, sub-threshold RDK stimuli are presented. The association between "top-down" and "bottom-up" activation will engage Hebbian-like learning mechanisms aiming to strengthen the response of hV5/MT+to sub-threshold stimuli. The hypothesis is that once these pathways are strengthened, the presentation of sub-threshold stimuli will elicit enough activity in area hV5/MT+to improve performance in the direction of motion discrimination task. We note that $h V 5 / M T+c a n$ be upregulated via rt-fMRI nFb enhanced imagery even when it has lost its V1 input. Once the subject learns how to upregulate hV5/MT+inside the magnet over the period of training, we hypothesize that s/he will also be able to transfer this learned voluntary ability during training outside the MRI environment.

maximize ipsi-lesional hV5/MT+activity using the imagery task. This $\mathrm{nFb}$ mediated, "topdown" increase in hV5/MT+activity will then be paired with the presentation of visual motion stimuli that are invisible (sub-threshold) to subjects with V1+lesions. We hypothesize that, by repeatedly presenting sub-threshold visual motion stimuli while hV5/MT+is activated in a "top-down" fashion by $\mathrm{nFb}$, we will engage Hebbian-like association learning mechanisms (Hebb, 1946; Rebesco and Miller, 2011; Gallistel and Matzel, 2013). These mechanisms will promote plasticity in the surviving, V1-bypassing pathways that become activated by the stimulus presentation and project to area hV5/MT+. In other words, we hypothesize that after $\mathrm{nFb}$ training, regions of area $\mathrm{hV} 5 / \mathrm{MT}+$ deprived of $\mathrm{V} 1$ input will respond more strongly to 
visual stimuli presented inside the scotoma, improving performance. If successful, this strategy will induce a "neural bypass" of V1 function with respect to visual motion perception.

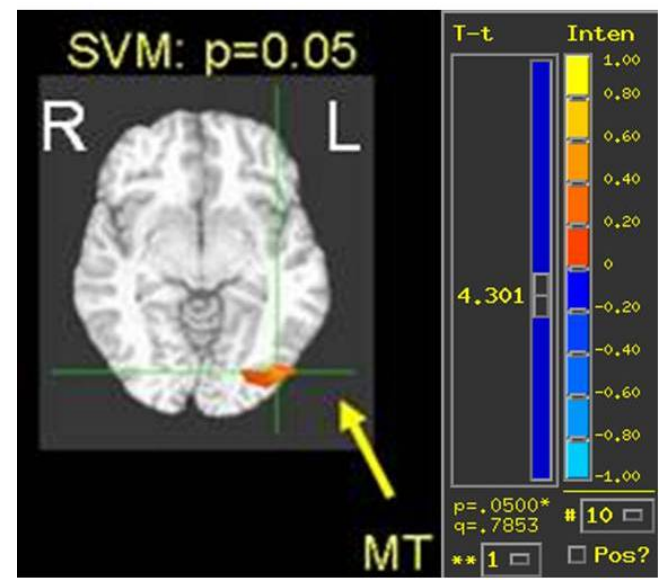

Figure 6. The support vector machine (SVM) algorithm defines area hV5/MT+when it is trained on coherent motion RDKS. Subjects fixated while two RDKs were presented simultaneously on symmetric locations in the right (red colorcoded activation) and left (blue color-coded activation) visual hemifields. The RDK presented in the left visual field was always kept at $0 \%$ coherence (no global motion direction), while the RDK in the right visual field alternated between $0 \%$ coherence and $100 \%$ coherence. A support vector machine algorithm was trained on the whole brain to classify when the coherent RDK (right visual field/red-color coded activation) was presented. The color map indicates the region that was important in classifying the presentation of a $100 \%$ coherent versus a $0 \%$ coherent RDK in the right visual field. As expected, the area underlined by the green crosshairs corresponds to area hV5/MT+. Five subjects tested gave consistent results (3dANOVA2 performed in AFNI, $\mathrm{p}=0.05$ ).

One advantage that $\mathrm{rt}-\mathrm{fMRI} \mathrm{nFb}$ methods have over behavioral feedback approaches is that specific brain pathways or areas can be selectively trained. It is then, feasible to train targeted components of the neural circuit that are likely to contribute to recovery. One example, is strengthening neural pathways that bypass the region of injury to promote recovery. Thus far, we have been discussing a univariate rt-fMRI $\mathrm{nFb}$ approach, where the $\mathrm{nFb}$ provided is proportional to the activity of a specific region of interest (in the above example, area hV5/MT + ) or, pattern of activity (Shibata et al., 2011). An alternative to the univariate $\mathrm{nFb}$ method is the multivariate classification approach (Papageorgiou et al., 2013; Papageorgiou et al., 2009; LaConte et al., 2007; Mourao-Miranda et al., 2005). In this approach, a classification algorithm, usually, the support vector machine (Vapnik, 1995) is trained on a set of relevant data in order to identify the brain networks that are involved in a specific computation (task). This is akin to the localizer that is used to identify the region of interest in the univariate approach. In fact, in the case of presentation of RDK stimuli, the multivariate SVM approach picks out chiefly area $\mathrm{hV} 5 / \mathrm{MT}+$, as expected (see fig. 6 below). However, in other cases, this approach may reveal different patterns of activity than expected, helping to formulate new hypotheses about the networks that might contribute to rehabilitation. Once the parameters of the classification algorithm are trained, the algorithm can be used to provide neuro-feedback to the subject in 
separate sessions. This approach may be effective in cases where the pathways that need to be modulated to induce recovery are not known a-priori.

(A) Healthy Subjects Group Maps ( $n=5)$

(A1) Right (red) vs. Left (blue) Direction of Motion Discrimination

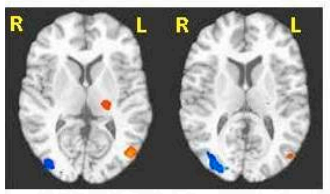

GLM: $p=0.01 ;$ FDR-corrected

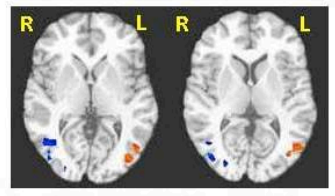

SVM: $p=0.05 ;$ FDR-corrected

(A2) SVM classifier output $75.6 \%$ accuracy

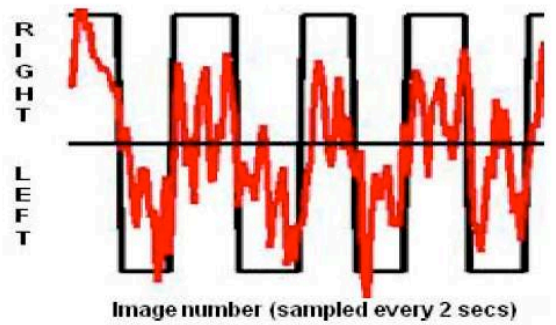

(B) Left Hemianopic Patient

(Right V1+ lesion)

(B1) hV5/MT+ Retinotopic Eccentricity Map of the left (normal) and right (lesioned) hemisphere with outlined hV5/MT+

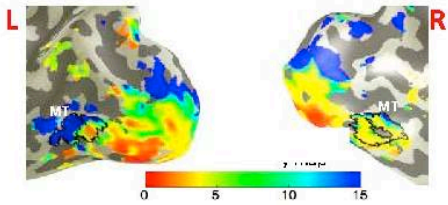

(B2) hV5/MT+ modulation using $\mathrm{rt}$-fMRI $\mathrm{nFb}$ training

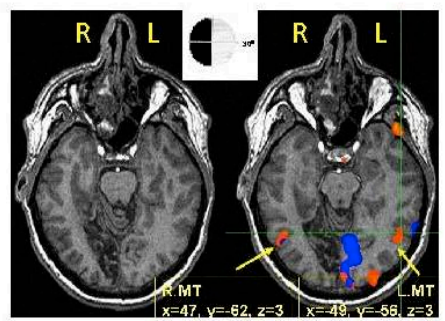

Figure 7. Rt-fMRI $n F b$ can be used to selectively upregulate hV5/MT+in a top-down manner during a mental imagery task. In the absence of a coherent moving stimulus, subjects $(n=5)$ were able to use imagery of coherent motion to selectively upregulate hV5/MT+via rt-fMRI nFb training. (A) Non-coherent RDKs were presented symmetrically in the Left $(L)$ and the $(R)$ hemifield and subjects were cued to imagine that the Lor the R RDK contained coherent motion. The level of contralateral hV5/MT+activity was delivered via the length of an arrow at the fixation point. (A1) Red/ blue regions correspond to voxels upregulated when the subject is instructed to imagine coherent motion in the right/left visual field, respectively. Similar results are obtained when using a standard GLM (left panel), versus plotting the weight vector of the support vector machine (SVM) algorithm that classifies whether subjects imagined coherent motion to the left versus the right hemifield (right panel). The regions identified correspond to area hV5/MT+in both hemispheres (color red, blue). Therefore, subjects can be trained via rt-fMRI nFb to modulate their hV5/MT+activity even in the absence of a coherently moving stimulus; (A2) Output (red curve) of a support vector machine classifier indicating the side of the visual field where the subject imagined coherent motion. The correct choice indicated by the black curve; the prediction of the classifier by the red curve. Positive values indicate the subject was instructed to imagine coherent motion on the right, negative on the left. Note that area hV5/MT+activity can predict the subject's perceptual state. (B1) Eccentricity maps of a subject with left hemianopia illustrating that area hV5/MT+(outlined) of the lesioned (right) hemisphrere can be visually driven. Only the posterior lateral aspect of the two inflated hemispheres is presented. (B2) Illustrates that $h V 5 / M T+c a n$ also be upregulated via rt-fMRI $n F b$ training in a V1+lesioned patient. Left Panel: Right (R) PCA lesion, resulting in left (L) hemianopia (inset). Right Panel: Red color-coded areas represent the activity elicited by the subject's coherent motion imagery in the left (hemianopic) visual field, while blue color-coded area represents non-coherent motion presentation, as generated by GLM. Left hemianopic visual field imagery of coherent motion activated hV5/MT+bilaterally. Preliminary data for implementing the proposal we outlined above are encouraging as they suggest that ipsilesional hV5/MT+activity: (1) conveys information about the stimulus (B1), and (2) can be upregulated using rt-fmri nFb imagery in $\mathrm{V} 1+$ lesioned patients (B2).

In summary, emerging strategies based on $\mathrm{rt}-\mathrm{fMRI} \mathrm{nFb}$ hold considerable promise, as they can be: 1) used to enhance plasticity in a number of systems (Berman et al., 2011; Bray et al., 2007; 
Caria et al., 2010; deCharms et al., 2004; Haller et al., 2010; Johnson et al., 2012; Johnston et al., 2010; Lee et al., 2012; Li et al., 2012; McCaig et al., 2011; Papageorgiou et al., 2013; Posse et al., 2003; Ruiz et al., 2013; Subramanian et al., 2011; Sulzer et al., 2013; Veit al., 2012; Yoo and Jolesz, 2002; Yoo et al., 2008; Zotev et al., 2011) including in the visual system of healthy participants (Scharnowski et al., 2012; Shibata et al., 2011); 2) superior to normal behavioral methods (deCharms et al., 2005); 3) tailored to induce perceptual learning (plasticity) in a highly specific fashion (Shibata et al., 2011); 4) used to identify pathways relevant to recovery via multivariate computational methods; and 5) used to induce long-lasting learning effects reported to persist outside the magnet, after the end of training (Ruiz et al., 2013; Sulzer et al., 2013). In the longterm, rtfMRI $\mathrm{nFb}$ methods promise to induce cortical plasticity that is efficient, robust and targeted for each patient. Lessons learned are likely to apply beyond the visual system to disorders of motor function, cognition, speech, language and emotion.

\section{Challenges and future considerations using rt-fMRI $\mathrm{nFb}$ for visual neurorehabilitation}

Many challenges need to be overcome in order to study the efficacy of rt-fMRI nFb methods in visual rehabilitation. Primarily, we need to develop effective rt-fMRI nFb paradigms in subjects with $\mathrm{V} 1$ lesions. The challenge is to implement rt-fMRI nFb training protocols to strengthen specific pathways that are hypothesized to play a role in visual performance. It is important to understand which visual pathways are more amenable to rehabilitation and what is the best rt-fMRI $\mathrm{nFb}$ paradigm to use. This requires elucidating which factors are "necessary and sufficient variables for learning" (Weiskopf, 2012). The answers to these questions will in general depend on the specifics of the visual function that requires rehabilitation, as well as on other factors such as the subject's motivation.

Quantifying the degree of induced reorganization using population receptive field ( $\mathrm{pRF}$ ) methods is complementary to behavioral performance measures and represents a valuable neuroimaging biomarker for studying the mechanism of recovery induced by $\mathrm{nFb}$ rehabilitation methods. Information obtained will then, allow us to refine future rehabilitative approaches. Several different pathways may be able to contribute to recovery. For example, in the case of the visual motion rehabilitation example, the focus was on strengthening "bottomup" pathways to enhance the response of the ipsilesional area hV5/MT+to the visual motion stimulus. One can hypothesize other strategies that focus instead on reorganizing higher areas, such as frontal eye fields (FEF), supplementary eye fields (SEF) areas involved in the generation of visual motion percepts downstream of hV5/MT+by "reading out" the weak activity that persists in extrastriate cortex following V1+lesions. Or, one can focus on strategies that reorganize attentional networks, such as middle frontal gyrus (mFG), intraparietal sulcus (IPS), superior parietal lobule (SPL), and anterior cingulate cortex (ACC) that enhance the weakened responses elicited in surviving areas following V1+lesions.

There are important technical challenges. One criticism is that rt-fMRI $\mathrm{nFb}$ approaches are impractical because they require large amounts of magnet time. Although this may have some truth in it, preliminary studies reveal that time spent inside the magnet is much less than what 
pure behavioral methods, require. Deciding how many rt-fMRI $\mathrm{nFb}$ sessions are needed to induce plasticity is a question that still needs to be answered. Preliminary evidence suggests that as few as 5-10 sessions can be sufficient to induce a strong perceptual learning effect in normal subjects (Shibata et al., 2011, and unpublished data of ours), but this will need to be validated specifically in patients with V1 lesions. Healthy participants and patients undergoing rt-fMRI $\mathrm{nFb}$ training sessions inside the magnet can learn to voluntarily elicit the desired pattern of activity. Subjects can then, gradually learn to implement this process outside the magnet, transferring their experience from rt-fMRI $\mathrm{nFb}$ sessions to ordinary behavioral sessions. We do not have adequate evidence yet, to determine under what conditions training accomplished inside the rt-fMRI environment can be transferred outside the magnet, but there is reason to be hopeful (Ruiz et al., 2013; Sulzer et al., 2013). The clinical applicability of rt-fMRI $\mathrm{nFb}$ training will become significantly broader if it becomes feasible to decouple the patient's training sessions from the rt-fMRI $\mathrm{nFb}$ environment. Another important question is how long the effects of $\mathrm{rt}-\mathrm{fMRI} \mathrm{nFb}$ training are expected to last and whether this depends on the number of rt-fMRI $\mathrm{nFb}$ sessions used for training. Here too, there is reason for optimism given the results of Shibata et al. who managed to induce perceptual learning within 5 sessions of rtfMRI $\mathrm{nFb}$ (Shibata et al., 2011).

Many important challenges remain. However, it is now possible to lay the foundation of a systematic approach to visual rehabilitation using novel $\mathrm{rt}-\mathrm{fMRI} \mathrm{nFb}$ methods guided by $\mathrm{pRF}$ analysis of spared visual areas. This approach promises to teach us a lot about the visual system's capacity for plasticity after injury, and offers hope that effective, and robust visual rehabilitation methods, such as the novel rt-fMRI $\mathrm{nFb}$ approach will be used in the field of visual neuro-rehabilitation.

\section{Conclusion}

Neurorehabiliation of visual loss that occurs as a result of primary visual cortex injury is a difficult problem. To date, we have little understanding of the plasticity and reorganization mechanisms operating in the adult visual system following V1 injury. Consequently, no reliable method exists to effectively rehabilitate V1-lesioned patients who experience loss of visual perception in the contralateral hemifield (Horton, 2005a; Horton, 2005b; Pambakian and Kennard, 1997). Interestingly, recent results have shown that visually driven activity persists in extrastriate cortex following chronic area V1+lesions (Schmid, 2010; Schmid, 2009; Rodman, 1989; Rodman, 1990; Baseler, 1999), and that it is possible in some cases to rehabilitate visual motion perception (Das, 2010; Huxlin, 2009; Raninen et al., 2007; Henriksson et al., 2007; Sahraie et al., 2010). This confirms the existence of functional pathways that bypass the V1+lesion, providing direct input to spared extrastriate cortex. Such pathways are generally too weak to result in practical benefit. However, appropriate training strategies may be able to strengthen them sufficiently to induce recovery.

Real-time fMRI neuro-feedback strategies allow subjects to voluntarily modulate activity in certain brain areas or, neural pathways. These methods can be used to promote plasticity (Shibata et al., 2011). For example, we hypothesize that rt-fMRI $\mathrm{nFb}$ may be used to strengthen pathways that bypass the region of V1 injury to transmit visual motion information to area 
hV5/MT+. One paradigm that could, in theory, be used to accomplish this is the following: Subjects are trained by rt-fMRI $\mathrm{nFb}$ to voluntarily upregulate their hV5/MT+activity. Whenever hV5/MT+activity crosses a pre-set threshold, sub-threshold visual stimuli are presented. Repeated pairing of the "top-down" nFb-driven activation with the "bottom-up" stimulusdriven activation will engage Hebbian-like, association learning mechanisms, strengthening the response of $\mathrm{hV} 5 / \mathrm{MT}+$ to visual motion stimuli. Visual motion stimuli that were previously sub-threshold may then, rise above threshold following training, improving performance.

Rehabilitating dense visual field scotomas requires adopting a systematic approach. Plasticity changes induced by new rehabilitation strategies should be mapped and their mechanism studied. We have presented evidence that $\mathrm{pRF}$ analysis is an excellent tool for this purpose, quantifying changes and providing rich data for formulating hypotheses about what regions of the visual field may be more amenable to rehabilitation and what pathways contribute to recovery.

We conclude that, even though rt-fMRI nFb methods are currently in their infancy, they hold considerable promise for inducing plasticity in targeted pathways promoting successful rehabilitation. Although here, we have focused on the visual system, principles discussed apply to the neuro-rehabilitation of several other domains of brain function, such as motor control, language, speech (Papageorgiou et al., 2009a; Papageorgiou et al., 2009b; Papageorgiou et al., 2013), emotion and cognition.

\section{Acknowledgements}

Part of the work that is presented here was supported by: (i) a McNair Foundation award, a McNair Medical Institute (MMI) award and a Fight for Sight Grant to T.D. Papageorgiou; and (ii) an NEI RO1 (EY019272), DoD (W81XWH-08-2-0146), and an HHMI Early Career Award to S. M. Smirnakis.

\section{Author details}

T. Dorina Papageorgiou ${ }^{1,2}$, Amalia Papanikolaou ${ }^{4}$ and Stelios M. Smirnakis ${ }^{1,3,4}$

1 Department of Neurology, Baylor College of Medicine, Houston, Texas, USA

2 Department of Physical Medicine and Rehabilitation, Baylor College of Medicine, Houston, Texas, USA

3 Deparment of Neuroscience, Baylor College of Medicine, Houston, Texas, USA

4 Departments of MaxPlanck Institute for Biological Cybernetics, Tuebingen, Germany 


\section{References}

[1] Ahissar, M. and S. Hochstein (1997). "Task difficulty and the specificity of perceptual learning." Nature 387(6631): 401-6.

[2] Ajina, S. and C. Kennard (2012). "Rehabilitation of damage to the visual brain." Rev Neurol (Paris) 168(10): 754-61.

[3] Alexander, I. and A. Cowey (2009). "The cortical basis of global motion detection in blindsight." Exp Brain Res 192(3): 407-11.

[4] Amano, K., B. A. Wandell, et al. (2009). "Visual field maps, population receptive field sizes, and visual field coverage in the human MT+ complex." J Neurophysiol 102(5): 2704-18.

[5] Anand, A., Y. Li, et al. (2007). "Reciprocal effects of antidepressant treatment on activity and connectivity of the mood regulating circuit: an FMRI study." J Neuropsychiatry Clin Neurosci 19(3): 274-82.

[6] Apkarian, A. V., M. C. Bushnell, et al. (2005). "Human brain mechanisms of pain perception and regulation in health and disease." Eur J Pain 9(4): 463-84.

[7] Baseler, H. A., A. Gouws, et al. (2011). "Large-scale remapping of visual cortex is absent in adult humans with macular degeneration." Nat Neurosci 14(5): 649-55.

[8] Baseler, H. A., A. B. Morland, et al. (1999). "Topographic organization of human visual areas in the absence of input from primary cortex." J Neurosci 19(7): 2619-27.

[9] Berman, B. D., S. G. Horovitz, et al. (2012). "Self-modulation of primary motor cortex activity with motor and motor imagery tasks using real-time fMRI-based neurofeedback." Neuroimage 59(2): 917-25.

[10] Boynton, G. M., S. A. Engel, et al. (1996). "Linear systems analysis of functional magnetic resonance imaging in human V1." J Neurosci 16(13): 4207-21.

[11] Bray, S., S. Shimojo, et al. (2007). "Direct instrumental conditioning of neural activity using functional magnetic resonance imaging-derived reward feedback." J Neurosci 27(28): 7498-507.

[12] Brown, T. H., P. F. Chapman, et al. (1988). "Long-term synaptic potentiation." Science 242(4879): 724-8.

[13] Caria, A., R. Sitaram, et al. (2010). "Volitional control of anterior insula activity modulates the response to aversive stimuli. A real-time functional magnetic resonance imaging study." Biol Psychiatry 68(5): 425-32.

[14] Caria, A., R. Veit, et al. (2007). "Regulation of anterior insular cortex activity using real-time fMRI." Neuroimage 35(3): 1238-46. 
[15] Censor, N. and D. Sagi (2009). "Global resistance to local perceptual adaptation in texture discrimination." Vision Res 49(21): 2550-6.

[16] Chiew, M., S. M. LaConte, et al. (2012). "Investigation of fMRI neurofeedback of differential primary motor cortex activity using kinesthetic motor imagery." Neuroimage 61(1): 21-31.

[17] Chokron, S., C. Perez, et al. (2008). "From blindsight to sight: cognitive rehabilitation of visual field defects." Restor Neurol Neurosci 26(4-5): 305-20.

[18] Corbetta, M. and G. L. Shulman (2002). "Control of goal-directed and stimulus-driven attention in the brain." Nat Rev Neurosci 3(3): 201-15.

[19] Cowey, A. (2010). "Visual system: how does blindsight arise?" Curr Biol 20(17): R702-4.

[20] Cowey, A. (1974). "Atrophy of retinal ganglion cells after removal of striate cortex in a rhesus monkey." Perception 3(3): 257-60.

[21] Cowey, A. and P. Stoerig (1997). Blindsight in man and monkey. Brain 120( Pt 3): 535-59.

[22] Cowey, A. and P. Stoerig (1989). "Projection patterns of surviving neurons in the dorsal lateral geniculate nucleus following discrete lesions of striate cortex: implications for residual vision." Exp Brain Res 75(3): 631-8.

[23] Cowey, A. and P. Stoerig (1991). "The neurobiology of blindsight." Trends Neurosci 14(4): 140-5.

[24] Cox, R. W. (1996). "AFNI: software for analysis and visualization of functional magnetic resonance neuroimages." Comput Biomed Res 29(3): 162-73.

[25] Das, A. and K. R. Huxlin (2010). "New approaches to visual rehabilitation for cortical blindness: outcomes and putative mechanisms." Neuroscientist 16(4): 374-87.

[26] de Gelder, B., J. S. Morris, et al. (2005). "Unconscious fear influences emotional awareness of faces and voices." Proc Natl Acad Sci U S A 102(51): 18682-7.

[27] deCharms, R. C., K. Christoff, et al. (2004). "Learned regulation of spatially localized brain activation using real-time fMRI." Neuroimage 21(1): 436-43.

[28] deCharms, R. C., F. Maeda, et al. (2005). "Control over brain activation and pain learned by using real-time functional MRI." Proc Natl Acad Sci U S A 102(51): 18626-31.

[29] Deco, G. and T. S. Lee (2004). "The role of early visual cortex in visual integration: a neural model of recurrent interaction." Eur J Neurosci 20(4): 1089-100.

[30] DeYoe, E. A., G. J. Carman, et al. (1996). "Mapping striate and extrastriate visual areas in human cerebral cortex." Proc Natl Acad Sci U S A 93(6): 2382-6. 
[31] Dineen, J., A. Hendrickson, et al. (1982). "Alterations of retinal inputs following striate cortex removal in adult monkey." Exp Brain Res 47(3): 446-56.

[32] Dougherty, R. F., M. Ben-Shachar, et al. (2005). "Functional organization of human occipital-callosal fiber tracts." Proc Natl Acad Sci U S A 102(20): 7350-5.

[33] Dougherty RF, V.M. Koch, et al. (2003). "Visual field representations and locations of visual areas V1/2/3 in human visual cortex." J Vis. 3(10):586-98.

[34] Dumoulin, S. O. and B. A. Wandell (2008). "Population receptive field estimates in human visual cortex." Neuroimage 39(2): 647-60.

[35] Elisevich, K. V., R. M. Ford, et al. (1984). "Visual abnormalities with multiple trauma." Surg Neurol 22(6): 565-75.

[36] Engel, S. A., D. E. Rumelhart, et al. (1994). "fMRI of human visual cortex." Nature 369(6481): 525.

[37] Fabre-Thorpe, M., F. Levesque, et al. (1994). "Preservation of pointing accuracy toward moving targets after extensive visual cortical ablations in cats." Cortex 30(4): 585-601.

[38] Fendrich, R., C. M. Wessinger, et al. (1992). "Residual vision in a scotoma: implications for blindsight." Science 258(5087): 1489-91.

[39] ffytche, D.H., A. Howseman, et al. (2000). Human area V5 and motion in the ipsilateral visual field. Eur J Neurosci. 12(8):3015-25.

[40] Fields, R. D. (2008). "White matter in learning, cognition and psychiatric disorders." Trends Neurosci 31(7): 361-70.

[41] Frank, S., S. Lee, et al. (2012). "The obese brain athlete: self-regulation of the anterior insula in adiposity." PLoS One 7(8): e42570.

[42] Friel, K. M., A. A. Heddings, et al. (2000). "Effects of postlesion experience on behavioral recovery and neurophysiologic reorganization after cortical injury in primates." Neurorehabil Neural Repair 14(3): 187-98.

[43] Fujii, T., H. C. Tanabe, et al. (2009). "An investigation of cross-modal plasticity of effective connectivity in the blind by dynamic causal modeling of functional MRI data." Neurosci Res 65(2): 175-86.

[44] Gall, C., B. Steger, et al. (2013). "Evaluation of two treatment outcome prediction models for restoration of visual fields in patients with postchiasmatic visual pathway lesions." Neuropsychologia.

[45] Gallistel, C. R. and L. D. Matzel (2013). "The neuroscience of learning: beyond the Hebbian synapse." Annu Rev Psychol 64: 169-200.

[46] Gilhotra, J. S., P. Mitchell, et al. (2002). "Homonymous visual field defects and stroke in an older population." Stroke 33(10): 2417-20. 
[47] Giorgi, R. G., R. L. Woods, et al. (2009). "Clinical and laboratory evaluation of peripheral prism glasses for hemianopia." Optom Vis Sci 86(5): 492-502.

[48] Girard, P., P. A. Salin, et al. (1991). "Visual activity in areas V3a and V3 during reversible inactivation of area V1 in the macaque monkey." J Neurophysiol 66(5): 1493-503.

[49] Goebel, R., L. Muckli, et al. (2001). "Sustained extrastriate cortical activation without visual awareness revealed by fMRI studies of hemianopic patients." Vision Res 41(10-11): 1459-74.

[50] Haller, S., N. Birbaumer, et al. (2010). "Real-time fMRI feedback training may improve chronic tinnitus." Eur Radiol 20(3): 696-703.

[51] Hamilton, J. P., G. H. Glover, et al. (2011). "Modulation of subgenual anterior cingulate cortex activity with real-time neurofeedback." Hum Brain Mapp 32(1): 22-31.

[52] Hebb, D.O. (1949). Distinction in coherent neural network between resting and working brain states. The organization of behavior. New York: Wiley \& Sons.

[53] Henriksson, L., A. Raninen, et al. (2007). "Training-induced cortical representation of a hemianopic hemifield." J Neurol Neurosurg Psychiatry 78(1): 74-81.

[54] Henry, S.H. R.C. Reid (2000). The koniocellular pathway in primate vision. Annual Review of Neuroscience 23: 127-153.

[55] Hinds, O., S. Ghosh, et al. (2011). "Computing moment-to-moment BOLD activation for real-time neurofeedback." Neuroimage 54(1): 361-8.

[56] Hinrichs, H., H. J. Heinze, et al. (2006). "Causal visual interactions as revealed by an information theoretic measure and fMRI." Neuroimage 31(3): 1051-60.

[57] Hopfinger, J. B., M. H. Buonocore, et al. (2000). "The neural mechanisms of top-down attentional control." Nat Neurosci 3(3): 284-91.

[58] Horton, J. C. (2005a). "Disappointing results from Nova Vision's visual restoration therapy." Br J Ophthalmol 89(1): 1-2.

[59] Horton, J. C. (2005b). "Vision restoration therapy: confounded by eye movements." $\mathrm{Br}$ J Ophthalmol 89(7): 792-4.

[60] Hua, T., P. Bao, et al. (2010). "Perceptual learning improves contrast sensitivity of V1 neurons in cats." Curr Biol 20(10): 887-94.

[61] Hubel, D. H. and Wiesel, T. N. (1977). Ferrier Lecture: Functional Architecture of Macaque Monkey Visual Cortex, Proceedings of the Royal Society of London. Series B, Biological Sciences, Vol. 198, No. 1130 (May 19, 1977), pp. 1-59 Published by: The Royal Society Stable URL: http://www.jstor.org/stable/77245.

[62] Huxlin, K. R., J. M. Williams, et al. (2008a). "A neurochemical signature of visual recovery after extrastriate cortical damage in the adult cat." J Comp Neurol 508(1): 45-61. 
[63] Huxlin, K. R. (2008b). "Perceptual plasticity in damaged adult visual systems." Vision Res 48(20): 2154-66.

[64] Huxlin, K. R., T. Martin, et al. (2009). "Perceptual relearning of complex visual motion after V1 damage in humans." J Neurosci 29(13): 3981-91.

[65] Huxlin, K. R. and T. Pasternak (2004). "Training-induced recovery of visual motion perception after extrastriate cortical damage in the adult cat." Cereb Cortex 14(1): 81-90.

[66] Huxlin, K. Plasticity of Visual Perception After Permanent Damage to the Adult Visual Cortex. in "Visual Field restoration Therapy Symposium" - Nanos Annual Meeting. 2006. Tuscon, AZ.

[67] Iorizzo, D. B., M. E. Riley, et al. (2011). "Differential impact of partial cortical blindness on gaze strategies when sitting and walking - an immersive virtual reality study." Vision Res 51(10): 1173-84.

[68] Jobke, S., E. Kasten, et al. (2009). "Vision restoration through extrastriate stimulation in patients with visual field defects: a double-blind and randomized experimental study." Neurorehabil Neural Repair 23(3): 246-55.

[69] Johnson, K. A., K. Hartwell, et al. (2012). "Intermittent "real-time" fMRI feedback is superior to continuous presentation for a motor imagery task: a pilot study." J Neuroimaging 22(1): 58-66.

[70] Johnston, S., D. E. Linden, et al. (2011). "Upregulation of emotion areas through neurofeedback with a focus on positive mood." Cogn Affect Behav Neurosci 11(1): 44-51.

[71] Johnston, S. J., S. G. Boehm, et al. (2010). "Neurofeedback: A promising tool for the self-regulation of emotion networks." Neuroimage 49(1): 1066-72.

[72] Julkunen, L., O. Tenovuo, et al. (2003). "Rehabilitation of chronic post-stroke visual field defect with computer-assisted training: a clinical and neurophysiological study." Restor Neurol Neurosci 21(1-2): 19-28.

[73] Karni, A. and D. Sagi (1991). "Where practice makes perfect in texture discrimination: evidence for primary visual cortex plasticity." Proc Natl Acad Sci U S A 88(11): 4966-70.

[74] Kasten, E., U. Bunzenthal, et al. (2007). "Vision restoration therapy does not benefit from costimulation: A pilot study." J Clin Exp Neuropsychol 29(6): 569-84.

[75] Kasten, E., U. Bunzenthal, et al. (2006). "Visual field recovery after vision restoration therapy (VRT) is independent of eye movements: an eye tracker study." Behav Brain Res 175(1): 18-26.

[76] Kasten, E., E. Muller-Oehring, et al. (2001). "Stability of visual field enlargements following computer-based restitution training - results of a follow-up." J Clin Exp Neuropsychol 23(3): 297-305. 
[77] Kasten, E., D. A. Poggel, et al. (1999). "Restoration of vision II: residual functions and training-induced visual field enlargement in brain-damaged patients." Restor Neurol Neurosci 15(2-3): 273-87.

[78] Kasten, E., D. A. Poggel, et al. (2000). "Computer-based training of stimulus detection improves color and simple pattern recognition in the defective field of hemianopic subjects." J Cogn Neurosci 12(6): 1001-12.

[79] Kasten, E. and B. A. Sabel (1995). "Visual field enlargement after computer training in brain-damaged patients with homonymous deficits: an open pilot trial." Restor Neurol Neurosci 8(3): 113-27.

[80] Kasten, E., S. Wuest, et al. (1998a). "Residual vision in transition zones in patients with cerebral blindness." J Clin Exp Neuropsychol 20(5): 581-98.

[81] Kasten, E., S. Wust, et al. (1998b). "Computer-based training for the treatment of partial blindness." Nat Med 4(9): 1083-7.

[82] Kastner, S. and L. G. Ungerleider (2000). "Mechanisms of visual attention in the human cortex." Annu Rev Neurosci 23: 315-41.

[83] Kerkhoff, G., U. Munssinger, et al. (1992). "Rehabilitation of homonymous scotomata in patients with postgeniculate damage of the visual system: saccadic compensation training." Restor Neurol Neurosci 4(4): 245-54.

[84] Kerkhoff, G., U. Munssinger, et al. (1994). "Neurovisual rehabilitation in cerebral blindness." Arch Neurol 51(5): 474-81.

[85] Kikuta, K., Y. Takagi, et al. (2006). "Early experience with 3-T magnetic resonance tractography in the surgery of cerebral arteriovenous malformations in and around the visual pathway." Neurosurgery 58(2): 331-7; discussion 331-7.

[86] Kluver, H. (1936). An analysis of the effects of the removal of the occipital lobes in monkeys, J Psychol, 2: 49- 61.

[87] LaConte, S. M., S. J. Peltier, et al. (2007). "Real-time fMRI using brain-state classification." Hum Brain Mapp 28(10): 1033-44.

[88] Law, C. T. and J. I. Gold (2008). "Neural correlates of perceptual learning in a sensory-motor, but not a sensory, cortical area." Nat Neurosci 11(4): 505-13.

[89] Lee, S., A. Papanikolaou, et al. (2013). "A new method for estimating population receptive field topography in visual cortex." Neuroimage 81: 144-57.

[90] Lee, S., S. Ruiz, et al. (2011). "Detection of cerebral reorganization induced by realtime fMRI feedback training of insula activation: a multivariate investigation." Neurorehabil Neural Repair 25(3): 259-67. 
[91] Lew, H. L., J. H. Poole, et al. (2007). "Program development and defining characteristics of returning military in a VA Polytrauma Network Site." J Rehabil Res Dev 44(7): 1027-34.

[92] Lewis, C. M., A. Baldassarre, et al. (2009). "Learning sculpts the spontaneous activity of the resting human brain." Proc Natl Acad Sci U S A 106(41): 17558-63.

[93] Li, X., K. J. Hartwell, et al. (2012). "Volitional reduction of anterior cingulate cortex activity produces decreased cue craving in smoking cessation: a preliminary realtime fMRI study." Addict Biol 18(4): 739-48.

[94] Lindberg, P., H. Forssberg, et al. (2003). "[Rehabilitation after stroke. Imaging techniques show how the cortical reorganization is affected by training]." Lakartidningen 100(51-52): 4289-92.

[95] Linden DE, Habes I, et al. (2012). Real-time self-regulation of emotion networks in patients with depression. PLoS One 7(6):e38115.

[96] Liu, Z. and D. Weinshall (2000). "Mechanisms of generalization in perceptual learning." Vision Res 40(1): 97- 109.

[97] Liu X, Zhu XH, Chen W. (2011). Distinction in coherent neural network between resting and working brain states. Brain Connect 1(5):377-88. doi: 10.1089/brain.2011.0044.

[98] Marshall, R. S., J. J. Ferrera, et al. (2008). "Brain activity associated with stimulation therapy of the visual borderzone in hemianopic stroke patients." Neurorehabil Neural Repair 22(2): 136-44.

[99] McCaig, R. G., M. Dixon, et al. (2011). "Improved modulation of rostrolateral prefrontal cortex using real-time fMRI training and meta-cognitive awareness." Neuroimage 55(3): 1298-305.

[100] McKenna, K., D. M. Cooke, et al. (2006). "The incidence of visual perceptual impairment in patients with severe traumatic brain injury." Brain Inj 20(5): 507-18.

[101] Mihailovic LT, Cupic D, Dekleva N. (1971). Changes in the numbers of neurons and glial cells in the lateral geniculate nucleus of the monkey during retrograde cell degeneration. J Comp Neurol 142: 223-9.

[102] Moore, T., H. R. Rodman, et al. (2001). "Direction of motion discrimination after early lesions of striate cortex (V1) of the macaque monkey." Proc Natl Acad Sci U S A 98(1): 325-30.

[103] Moore, T., H. R. Rodman, et al. (1995). "Localization of visual stimuli after striate cortex damage in monkeys: parallels with human blindsight." Proc Natl Acad Sci U S A 92(18): 8215-8.

[104] Morris, J. S., B. DeGelder, et al. (2001). "Differential extrageniculostriate and amygdala responses to presentation of emotional faces in a cortically blind field." Brain 124(Pt 6): 1241-52. 
[105] Mourao-Miranda, J., A. L. Bokde, et al. (2005). "Classifying brain states and determining the discriminating activation patterns: Support Vector Machine on functional MRI data." Neuroimage 28(4): 980-95.

[106] Mumford, D. (1994). Neuronal architectures for pattern theoretic problems. In: Large Scale Neuronal Theories of the Brain, Koch C, Davis, JL, eds., MIT Press, pp. 125-152.

[107] Okada, T., Y. Miki, et al. (2007). "Diffusion tensor fiber tractography for arteriovenous malformations: quantitative analyses to evaluate the corticospinal tract and optic radiation." AJNR Am J Neuroradiol 28(6): 1107-13.

[108] Olshausen, B.A. Principles of Image Representation in Visual Cortex. In: The Visual Neurosciences, L.M. Chalupa, J.S. Werner, Eds. MIT Press, 2003: 1603-1615.

[109] Overgaard, M. (2011). "Visual experience and blindsight: a methodological review." Exp Brain Res 209(4): 473-9.

[110] Pambakian, A.L. and C. Kennard (1997). Can visual function be restored in patients with homonymous hemianopia? Br J Ophthalmol (4): p. 324-8.

[111] Papageorgiou, T. D., W. A. Curtis, et al. (2009a). "Neurofeedback of two motor functions using supervised learning-based real-time functional magnetic resonance imaging." Conf Proc IEEE Eng Med Biol Soc 2009: 5377-80.

[112] Papageorgiou, T.D., M. McHenry, J.L. Lisinski, J.P. White, and S.M. LaConte (2009b). Speech rate control using supervised learning-based rea-time fMRI. 15th Annual Meeting of the Organization for Human Brain Mapping, San Franscisco. Neuroimage, 47: p. 97.

[113] Papageorgiou, T.D., LaConte, S.M., Lisinski, J.L., Peng, X., and Smirnakis, S.M, Classification Accuracy and Spatial Networks on Motion Coherence using Real-time fMRI. Hum Brain Mapp, Barcelona, 2010.

[114] Papageorgiou, T. D., J. M. Lisinski, et al. (2013). "Brain-computer interfaces increase whole-brain signal to noise." Proc Natl Acad Sci U S A 110(33): 13630-5.

[115] Papanikolaou, A., G.A. Keliris, T.D. Papageorgiou, Y. Shao, E. Krapp, E. Papageorgiou, K. Stingl, A. Bruckmann, U. Schiefer, N.K. Logothetis, and S.M. Smirnakis (2014). Population receptive field analysis of the primary visual cortex complements perimetry in patients with homonymous visual field defects. Proc Natl Acad Sci U S A 111(16):E1656-65.

[116] Pasik, T. and P. Pasik (1971). "The visual world of monkeys deprived of striate cortex: effective stimulus parameters and the importance of the accessory optic system." $V i$ sion Res Suppl 3: 419-35.

[117] Pessoa, L., S. Kastner, et al. (2003). "Neuroimaging studies of attention: from modulation of sensory processing to top-down control." J Neurosci 23(10): 3990-8. 
[118] Peyron, R., B. Laurent, et al. (2000). "Functional imaging of brain responses to pain. A review and meta-analysis (2000)." Neurophysiol Clin 30(5): 263-88.

[119] Pleger, B., A. F. Foerster, et al. (2003). "Functional magnetic resonance imaging mirrors recovery of visual perception after repetitive tachistoscopic stimulation in patients with partial cortical blindness." Neurosci Lett 335(3): 192-6.

[120] Plow, E. B., S. N. Obretenova, et al. (2011). "Combining visual rehabilitative training and noninvasive brain stimulation to enhance visual function in patients with hemianopia: a comparative case study." PM R 3(9): 825-35.

[121] Poggel, D. A., E. Kasten, et al. (2006). "Improving residual vision by attentional cueing in patients with brain lesions." Brain Res 1097(1): 142-8.

[122] Poggel, D. A., E. Kasten, et al. (2001). "Unusual spontaneous and training induced visual field recovery in a patient with a gunshot lesion." J Neurol Neurosurg Psychiatry 70(2): 236-9.

[123] Poggel, D. A., E. Kasten, et al. (2004). "Attentional cueing improves vision restoration therapy in patients with visual field defects." Neurology 63(11): 2069-76.

[124] Poggel, D. A., I. Mueller, et al. (2010). "Subjective and objective outcome measures of computer-based vision restoration training." NeuroRehabilitation 27(2): 173-87.

[125] Poppel, E., R. Held, et al. (1973). "Leter: Residual visual function after brain wounds involving the central visual pathways in man." Nature 243(5405): 295-6.

[126] Posse, S., D. Fitzgerald, et al. (2003). "Real-time fMRI of temporolimbic regions detects amygdala activation during single-trial self-induced sadness." Neuroimage 18(3): $760-8$.

[127] Raninen, A., S. Vanni, et al. (2007). "Temporal sensitivity in a hemianopic visual field can be improved by long-term training using flicker stimulation." J Neurol Neurosurg Psychiatry 78(1): 66-73.

[128] Rebesco, J. M. and L. E. Miller (2011). "Stimulus-driven changes in sensorimotor behavior and neuronal functional connectivity application to brain-machine interfaces and neurorehabilitation." Prog Brain Res 192: 83-102.

[129] Reinges, M. H., T. Krings, et al. (2004). "Functional and diffusion-weighted magnetic resonance imaging for visualization of the postthalamic visual fiber tracts and the visual cortex." Minim Invasive Neurosurg 47(3): 160-4.

[130] Reinhard, J., A. Schreiber, et al. (2005). "Does visual restitution training change absolute homonymous visual field defects? A fundus controlled study." Br J Ophthalmol 89(1): 30-5.

[131] Riggs, R. V., K. Andrews, et al. (2007). "Visual deficit interventions in adult stroke and brain injury: a systematic review." Am J Phys Med Rehabil 86(10): 853-60. 
[132] Rizzo, M. and D. A. Robin (1996). "Bilateral effects of unilateral visual cortex lesions in human." Brain 119( Pt 3): 951-63.

[133] Rodman, H. R., C. G. Gross, et al. (1989). "Afferent basis of visual response properties in area MT of the macaque. I. Effects of striate cortex removal." J Neurosci 9(6): 2033-50.

[134] Rodman, H. R., C. G. Gross, et al. (1990). "Afferent basis of visual response properties in area MT of the macaque. II. Effects of superior colliculus removal." J Neurosci 10(4): 1154-64.

[135] Rota, G., R. Sitaram, et al. (2009). "Self-regulation of regional cortical activity using real-time fMRI: the right inferior frontal gyrus and linguistic processing." Hum Brain Mapp 30(5): 1605-14.

[136] Rudolph, K. and T. Pasternak (1999). "Transient and permanent deficits in motion perception after lesions of cortical areas MT and MST in the macaque monkey." Cereb Cortex 9(1): 90-100.

[137] Rudolph, K. K., V. P. Ferrera, et al. (1994). "A reduction in the number of directionally selective neurons extends the spatial limit for global motion perception." Vision Res 34(24): 3241-51.

[138] Rudolph, T. M. and E. R. Delay (1993). "Recovery of a temporally based visual discrimination after visual cortex lesion in the rat." Behav Brain Res 53(1-2): 189-99.

[139] Ruiz, S., K. Buyukturkoglu, et al. (2013). "Real-time fMRI brain computer interfaces: Self-regulation of single brain regions to networks." Biol Psychol.

[140] Sabel, B. A., P. Henrich-Noack, et al. (2011). "Vision restoration after brain and retina damage: the "residual vision activation theory"." Prog Brain Res 192: 199-262.

[141] Sabel, B. A. and E. Kasten (2000). "Restoration of vision by training of residual functions." Curr Opin Ophthalmol 11(6): 430-6.

[142] Sabel, B. A., S. Kenkel, et al. (2004). "Vision restoration therapy (VRT) efficacy as assessed by comparative perimetric analysis and subjective questionnaires." Restor Neurol Neurosci 22(6): 399-420.

[143] Sahraie, A., M. J. Macleod, et al. (2010). "Improved detection following Neuro-Eye Therapy in patients with post-geniculate brain damage." Exp Brain Res 206(1): 25-34.

[144] Sahraie, A., C. T. Trevethan, et al. (2006). "Increased sensitivity after repeated stimulation of residual spatial channels in blindsight." Proc Natl Acad Sci U S A 103(40): 14971-6.

[145] Schmid, M. C., S. W. Mrowka, et al. (2010). "Blindsight depends on the lateral geniculate nucleus." Nature 466(7304): 373-7. 
[146] Schmid, M. C., T. Panagiotaropoulos, et al. (2009). "Visually driven activation in macaque areas V2 and V3 without input from the primary visual cortex." PLoS One 4(5): e5527.

[147] Schoenfeld, M. A., H. J. Heinze, et al. (2002). "Unmasking motion-processing activity in human brain area V5/MT+ mediated by pathways that bypass primary visual cortex." Neuroimage 17(2): 769-79.

[148] Schoth, F., U. Burgel, et al. (2006). "Diffusion tensor imaging in acquired blind humans." Neurosci Lett 398(3): 178-82.

[149] Schoups, A., R. Vogels, et al. (2001). "Practising orientation identification improves orientation coding in V1 neurons." Nature 412(6846): 549-53.

[150] Sereno, M. I., A. M. Dale, et al. (1995). "Borders of multiple visual areas in humans revealed by functional magnetic resonance imaging." Science 268(5212): 889-93.

[151] Scharnowski, F., C. Hutton, et al. (2012). "Improving visual perception through neurofeedback." J Neurosci 32(49): 17830-41.

[152] Sherbondy, A. J., R. F. Dougherty, et al. (2008). "Identifying the human optic radiation using diffusion imaging and fiber tractography." J Vis 8(10): 12 1-11.

[153] Shibata K, L.H. Chang, (2012). Decoding reveals plasticity in V3A as a result of motion perceptual learning. PLoS One. 7(8):e44003.

[154] Shibata, K., T. Watanabe, et al. (2011). "Perceptual learning incepted by decoded fMRI neurofeedback without stimulus presentation." Science 334(6061): 1413-5.

[155] Stilla, R., R. Hanna, et al. (2008). "Neural processing underlying tactile microspatial discrimination in the blind: a functional magnetic resonance imaging study." $J$ Vis 8(10): 13 1-19.

[156] Stoerig, P. and E. Barth (2001). "Low-level phenomenal vision despite unilateral destruction of primary visual cortex." Conscious Cogn 10(4): 574-87.

[157] Subramanian, L., J. V. Hindle, et al. (2011). "Real-time functional magnetic resonance imaging neurofeedback for treatment of Parkinson's disease." J Neurosci 31(45): 16309-17.

[158] Sulzer, J., R. Sitaram, et al. (2013). "Neurofeedback-mediated self-regulation of the dopaminergic midbrain." Neuroimage 83C: 817-825.

[159] Stoerig, P. and A. Cowey (1997). "Blindsight in man and monkey." Brain 120 ( Pt 3): 535-59.

[160] Takeuchi, N., T. Chuma, et al. (2005). "Repetitive transcranial magnetic stimulation of contralesional primary motor cortex improves hand function after stroke." Stroke 36(12): 2681-6. 
[161] Taoka, T., M. Sakamoto, et al. (2005). "Diffusion tensor imaging in cases with visual field defect after anterior temporal lobectomy." AJNR Am J Neuroradiol 26(4): 797-803.

[162] Taub, E., G. Uswatte, et al. (2002). "New treatments in neurorehabilitation founded on basic research." Nat Rev Neurosci 3(3): 228-36.

[163] Taylor, T. N. (1997). "The medical economics of stroke." Drugs 54 Suppl 3: 51-7; discussion 57-8.

[164] Van den Stock, J., M. Tamietto, et al. (2011). "Cortico-subcortical visual, somatosensory, and motor activations for perceiving dynamic whole-body emotional expressions with and without striate cortex (V1)." Proc Natl Acad Sci U S A 108(39): 16188-93.

[165] Vanburen, J. M. (1963). "Trans-Synaptic Retrograde Degeneration in the Visual System of Primates." J Neurol Neurosurg Psychiatry 26: 402-9.

[166] Vapnik, N. (1995). The nature of statistical learning theory. Springer.

[167] Veit, R., V. Singh, et al. (2012). "Using real-time fMRI to learn voluntary regulation of the anterior insula in the presence of threat-related stimuli." Soc Cogn Affect Neurosci 7(6): 623-34.

[168] Wandell, B. A., S. O. Dumoulin, et al. (2007). "Visual field maps in human cortex." Neuron 56(2): 366-83.

[169] Wang, L., W. Dai, et al. (2012). "Amplitude of low-frequency oscillations in first-episode, treatment-naive patients with major depressive disorder: a resting-state functional MRI study." PLoS One 7(10): e48658.

[170] Wedeen, V. J., D. L. Rosene, et al. (2012). "The geometric structure of the brain fiber pathways." Science 335(6076): 1628-34.

[171] Weiller, C. (1998). "Imaging recovery from stroke." Exp Brain Res 123(1-2): 13-7.

[172] Weiskopf, N. (2012). "Real-time fMRI and its application to neurofeedback." Neuroimage 62(2): 682-92.

[173] Weiskopf, N., R. Sitaram, et al. (2007). "Real-time functional magnetic resonance imaging: methods and applications." Magn Reson Imaging 25(6): 989-1003.

[174] Weiskopf, N., F. Sharnowski, et al., (2004). Self-regulation of local brain activity using real-time functional magnetic resonance imaging (fMRI). J Physiol Paris, 98(4-6): p. 357-73.

[175] Weiskopf, N., R. Veit, et al. (2003). "Physiological self-regulation of regional brain activity using real-time functional magnetic resonance imaging (fMRI): methodology and exemplary data." Neuroimage 19(3): 577-86.

[176] Weiskrantz, L. (2004). "Roots of blindsight." Prog Brain Res 144: 229-41. 
[177] Weiskrantz, L., E. K. Warrington, et al. (1974). "Visual capacity in the hemianopic field following a restricted occipital ablation." Brain 97(4): 709-28.

[178] Werth, R. and M. Moehrenschlager (1999). "The development of visual functions in cerebrally blind children during a systematic visual field training." Restor Neurol Neurosci 15(2-3): 229-41.

[179] Worsley, K. J., C. H. Liao, et al. (2002). "A general statistical analysis for fMRI data." Neuroimage 15(1): 1-15.

[180] Yang, T. and J. H. Maunsell (2004). "The effect of perceptual learning on neuronal responses in monkey visual area V4." J Neurosci 24(7): 1617-26.

[181] Yeatman, J. D., R. F. Dougherty, et al. (2012). "Tract profiles of white matter properties: automating fiber-tract quantification." PLoS One 7(11): e49790.

[182] Yoo, S. S. and F. A. Jolesz (2002). "Functional MRI for neurofeedback: feasibility study on a hand motor task." Neuroreport 13(11): 1377-81.

[183] Yoo, S. S., J. H. Lee, et al. (2008). "Neurofeedback fMRI-mediated learning and consolidation of regional brain activation during motor imagery." Int J Imaging Syst Technol 18(1): 69-78.

[184] Yotsumoto, Y., T. Watanabe, et al. (2008). "Different dynamics of performance and brain activation in the time course of perceptual learning." Neuron 57(6): 827-33.

[185] Zeki, S. (2004) Thirty years of a very special visual area, area V5. J. Physiol. 557, 1-2.

[186] Zeki S., and D.H. ffytche (1998). The Riddoch syndrome: insights into the neurobiology of conscious vision. Brain 121( Pt 1):25-45.

[187] Zhang, X., S. Kedar, et al. (2006). "Natural history of homonymous hemianopia." Neurology 66(6): 901-5.

[188] Zihl, J. and D. von Cramon (1979). "Restitution of visual function in patients with cerebral blindness." J Neurol Neurosurg Psychiatry 42(4): 312-22.

[189] Zihl, J. and D. von Cramon (1985). "Visual field recovery from scotoma in patients with postgeniculate damage. A review of 55 cases." Brain 108 ( Pt 2): 335-65.

[190] Zotev, V., F. Krueger, et al. (2011). "Self-regulation of amygdala activation using realtime FMRI neurofeedback." PLoS One 6(9): e24522.

[191] Zuiderbaan, W., B. M. Harvey, et al. (2012). "Modeling center-surround configurations in population receptive fields using fMRI." J Vis 12(3): 10. 